\title{
Genome-wide classification and expression analysis of MYB transcription factor families in rice and Arabidopsis
}

\author{
Amit Katiyar ${ }^{1,2}$, Shuchi Smita ${ }^{1,2}$, Sangram Keshari Lenka ${ }^{1,3}$, Ravi Rajwanshi ${ }^{1,4}$, Viswanathan Chinnusamy ${ }^{5}$
} and Kailash Chander Bansal ${ }^{1,2^{*}}$

\begin{abstract}
Background: The MYB gene family comprises one of the richest groups of transcription factors in plants. Plant MYB proteins are characterized by a highly conserved MYB DNA-binding domain. MYB proteins are classified into four major groups namely, 1R-MYB, 2R-MYB, 3R-MYB and 4R-MYB based on the number and position of MYB repeats. MYB transcription factors are involved in plant development, secondary metabolism, hormone signal transduction, disease resistance and abiotic stress tolerance. A comparative analysis of MYB family genes in rice and Arabidopsis will help reveal the evolution and function of MYB genes in plants.
\end{abstract}

Results: A genome-wide analysis identified at least 155 and 197 MYB genes in rice and Arabidopsis, respectively. Gene structure analysis revealed that MYB family genes possess relatively more number of introns in the middle as compared with C- and N-terminal regions of the predicted genes. Intronless MYB-genes are highly conserved both in rice and Arabidopsis. MYB genes encoding R2R3 repeat MYB proteins retained conserved gene structure with three exons and two introns, whereas genes encoding R1R2R3 repeat containing proteins consist of six exons and five introns. The splicing pattern is similar among R1R2R3 MYB genes in Arabidopsis. In contrast, variation in splicing pattern was observed among R1R2R3 MYB members of rice. Consensus motif analysis of $1 \mathrm{~kb}$ upstream region ( $5^{\prime}$ to translation initiation codon) of MYB gene ORFs led to the identification of conserved and over-represented cis-motifs in both rice and Arabidopsis. Real-time quantitative RT-PCR analysis showed that several members of MYBs are up-regulated by various abiotic stresses both in rice and Arabidopsis.

Conclusion: A comprehensive genome-wide analysis of chromosomal distribution, tandem repeats and phylogenetic relationship of MYB family genes in rice and Arabidopsis suggested their evolution via duplication. Genome-wide comparative analysis of MYB genes and their expression analysis identified several MYBs with potential role in development and stress response of plants.

\section{Background}

Transcription factors are essential regulators of gene transcription and usually consist of at least two domains namely a DNA-binding and an activation/repression domain, that function together to regulate the target gene expression [1]. The MYB (myeloblastosis) transcription factor family is present in all eukaryotes. "Oncogene"

\footnotetext{
* Correspondence: kailashbansal@hotmail.com

${ }^{1}$ National Research Centre on Plant Biotechnology, Indian Agricultural Research Institute, New Delhi 110012, India

${ }^{2}$ National Bureau of Plant Genetic Resources, Indian Agricultural Research Institute Campus, New Delhi 110012, India

Full list of author information is available at the end of the article
}

$v-M Y B$ was the first $M Y B$ gene identified in avian myeloblastosis virus [2]. Three $v-M Y B$-related genes namely c- $M Y B, A-M Y B$ and B- $M Y B$ were subsequently identified in many vertebrates and implicated in the regulation of cell proliferation, differentiation, and apoptosis [3]. Homologous genes were also identified in insects, fungi and slime molds [4]. A homolog of mammalian c-MYB gene, Zea mays $C 1$, involved in regulation of anthocyanin biosynthesis, was the first $M Y B$ gene to be characterized in plants [5]. Interestingly, plants encode large number of $M Y B$ genes as compared to fungi and animals [6-12]. MYB proteins contain a MYB DNA-binding domain, which is approximately 52 amino acid residues in length, 
and forms a helix-turn-helix fold with three regularly spaced tryptophan residues [13]. The three-dimensional structure of the MYB domain showed that the DNA recognition site $\alpha$-helix interacts with the major groove of DNA [14]. However, amino acid sequences outside the MYB domain are highly divergent. Based on the number of adjacent MYB repeats, MYB transcription factors are classified into four major groups, namely $1 \mathrm{R}-\mathrm{MYB}$, 2R-MYB, 3R-MYB and 4R-MYB containing one, two, three and four MYB repeats, respectively. In animals, R1R2R3type MYB domain proteins are predominant, while in plants, the R2R3-type MYB domain proteins are more prevalent $[4,7,15]$. The plant R2R3-MYB genes probably evolved from an R1R2R3-MYB gene progenitor through loss of R1 repeat or from an R1-MYB gene through duplication of R1 repeat [16,17].

In plants, $M Y B$ transcription factors play a key role in plant development, secondary metabolism, hormone signal transduction, disease resistance and abiotic stress tolerance $[18,19]$. Several R2R3-MYB genes are involved in regulating responses to environmental stresses such as drought, salt, and cold $[9,20]$. Transgenic rice over expressing OsMYB3R-2 exhibited enhanced cold tolerance as well as increased cell mitotic index [21]. Enhanced freezing stress tolerance was observed in Arabidopsis over-expressing OsMYB4 [10,22]. Arabidopsis AtMYB96, an R2R3-type $M Y B$ transcription factor, regulates drought stress response by integrating ABA and auxin signals [23]. Transgenic Arabidopsis expressing AtMYB15 exhibited hypersensitivity to exogenous ABA and improved tolerance to drought [24], and cold stress [20]. The AtMYB15 negatively regulated the expression of $C B F$ genes and conferred freezing tolerance in Arabidopsis [20]. Other functions of MYBs include control of cellular morphogenesis, regulation of secondary metabolism, meristem formation and the cell cycle regulation [15,25-28]. Recent studies have shown that the MYB genes are posttranscriptionally regulated by microRNAs; for instance, AtMYB33, AtMYB35, AtMYB65 and AtMYB101 genes involved in anther or pollen development are targeted by miR159 family $[29,30]$.

$M Y B$ TF family genes have been identified in a number of monocot and dicot plants [9], and evolutionary relationship between rice and Arabidopsis MYB proteins has been reported [31]. We report here genome-wide classification of 155 and 197 MYB TF family genes in rice and Arabidopsis, respectively. We also analysed abiotic stress responsive and tissue specific expression pattern of the selected $M Y B$ genes. To map the evolutionary relationship among $M Y B$ family members, phylogenetic trees were constructed for both rice and Arabidopsis MYB proteins. Several over- represented cis-regulatory motifs in the promoter region of the $M Y B$ genes were also identified.

\section{Results and discussion}

Identification, classification and structural analysis of MYB family members

Genome-wide analysis led to the identification of 155 and 197 MYB genes in rice and Arabidopsis, respectively, with their mapping on different chromosomes (Additional file 1: Table S1). We used previously assigned names to the MYB genes; for instance, AtMYBO (GL1) name was accepted for the first identified R2R3 $M Y B$ gene; subsequently identified R2R3 $M Y B$ genes were named as AtMYB1, AtMYB2, etc. in Arabidopsis [31-34]. We classified $M Y B$ transcription factors in to four distinct groups namely "MYB-related genes", "MYBR2R3", "MYB-R1R2R3", and "Atypical MYB genes" based on the presence of one, two, three and four MYB repeats, respectively. Our analysis revealed that the MYB-R2R3 subfamily consisted of the highest number of $M Y B$ genes, with 56.77 and $70.05 \%$ of the total $M Y B$ genes in rice and Arabidopsis, respectively (Figure 1a, b). In the R2R3MYB proteins, N-terminal consists of MYB domains, while the regulatory $\mathrm{C}$-terminal region is highly variable. Presence of a single MYB-like domain (e.g. hTRF1/ hTRF2) in their $\mathrm{C}$ terminus is required for telomeric DNA binding in vitro [35]. Earlier study revealed that the R2R3-MYB related proteins arose after loss of the sequences encoding $\mathrm{R} 1$ in an ancestral $3 \mathrm{R}-M Y B$ gene during plant evolution [36]. In contrast, only few $M Y B$ R1R2R3 genes were identified in Arabidopsis and rice with 5 and 4 genes, respectively. The category "MYBrelated genes" usually but not always contain a single MYB domain $[17,31,36]$. We found that "MYB-related genes" represented 40 and $26.39 \%$ of the total $M Y B$ genes in rice and Arabidopsis, respectively (Figure 1a, b), and thus constituted the second largest group of MYB proteins in both rice and Arabidopsis. We also identified one MYB protein in rice and two MYB proteins in Arabidopsis that contained more than three MYB repeats and these belong to "Atypical MYB genes" group. The AT1G09770 in Arabidopsis and LOC_Os07g04700 in rice have five MYB domains and are called as CDC5-type protein, whereas AT3G18100 of Arabidopsis has four MYB domains and is named as 4R-type MYB (Table 1; Additional file 1: Table S1). The 4R-MYB proteins belong to the smallest class, which contains R1/R2-like repeats. $M Y B$ genes can also be classified into several subgroups based on gene function, such as Circadian Clock Associated1 (CCA1) and Late Elongated Hypocotyl (LHY), Triptychon (TRY) and Caprice (CPC) $[15,17,37]$. $\mathrm{CPC}$ and TRY belong to the R3-MYB group and are mainly involved in epidermal cell differentiation, together with ENHANCER OF TRY AND CPC1, 2 and 3 (ETC1, ETC2 and ETC3), and TRICHOMELESS1 and 2 (TCL1 and TCL2) [38-41]. Here, we observed that CCA1, CPC and LHY subgroups contain 23, 3 and 1 'MYB-related' 


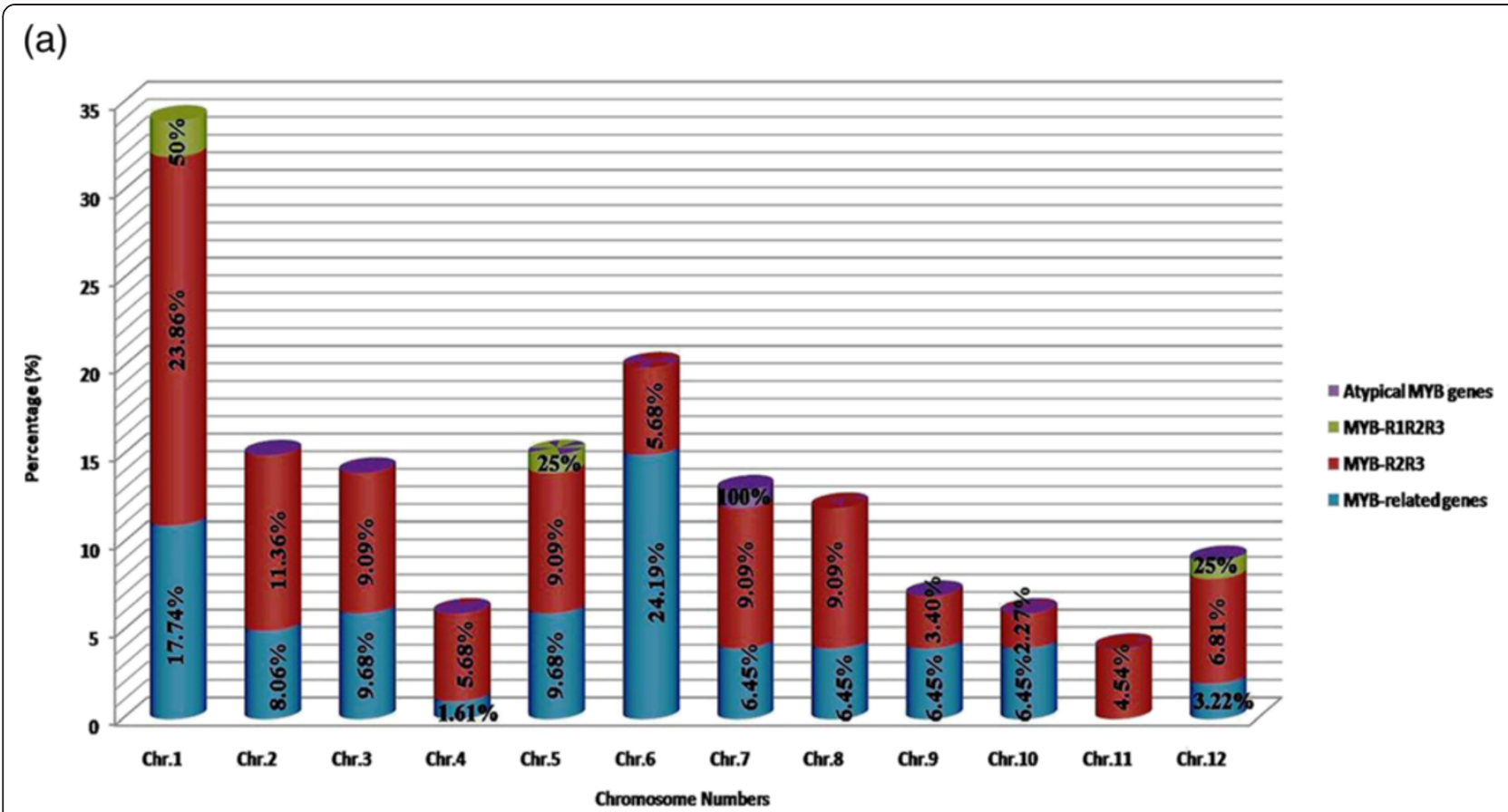

(b)

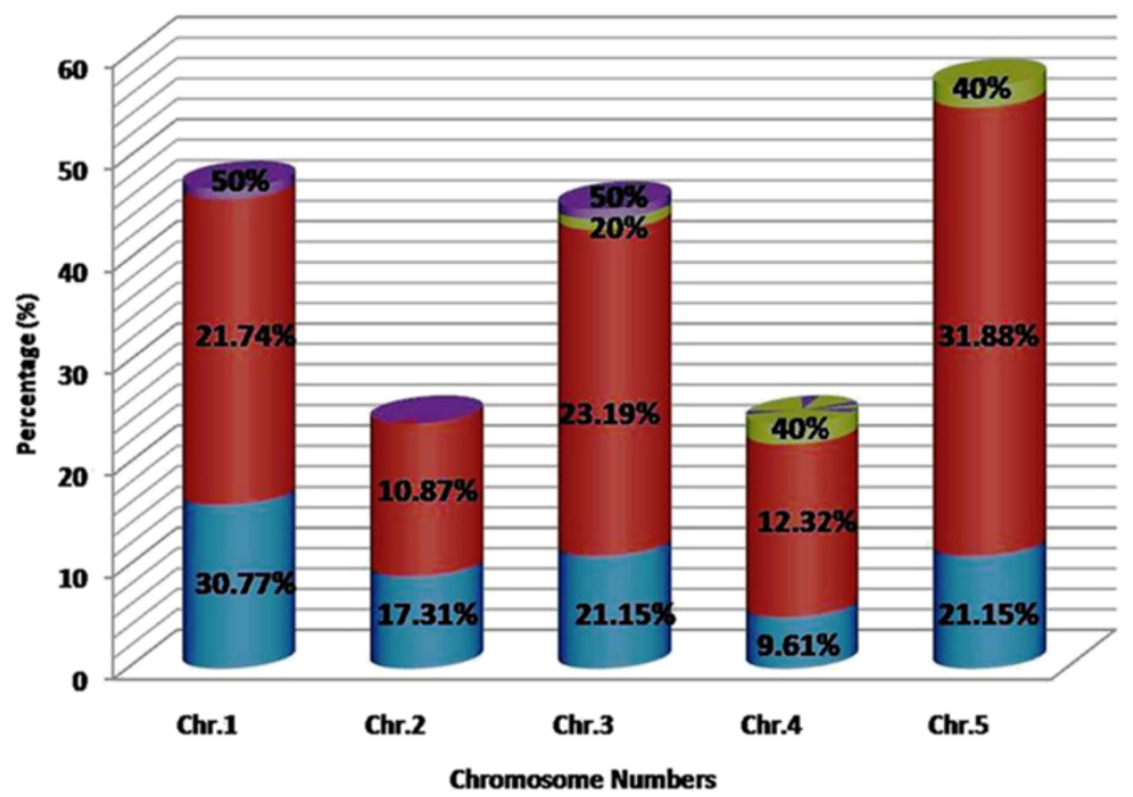

\section{Atypical MYB genes I MYB-R1R2R3 \\ E MYB-R2R3 \\ m MYB-relatedgenes}

Figure 1 Chromosome-wise distribution of MYB transcription factor genes. a) rice, b) Arabidopsis. We classified MYB transcription factors in to four distinct groups namely "MYB-related genes", "MYB-R2R3", "MYB-R1R2R3", and "Atypical MYB genes" based on the presence of one, two, three and four MYB repeats, respectively.

TF, respectively in Arabidopsis. To further understand the nature of MYB proteins, their physiochemical properties were also analyzed. The MYB proteins have similar grand average hydropathy (GRAVY) scores. Kyte and Doolittle [42] proposed that higher average hydropathy score of a protein indicates physiochemical property of an integral membrane protein, while a negative score indicates soluble nature of the protein. We observed that all MYB proteins in rice and Arabidopsis, except AT1G35516 had a negative GRAVY score, suggesting that MYBs are soluble proteins, a character that is necessary for transcription factors. Minimum and maximum score of GRAVY were recorded as -1.287 (LOC_Os02g47744) and -0.178 (LOC_Os08g37970) in rice, and -1.359 
Table 1 MYB-domain based characterization and comparison of MYB transcription factor family genes in terms of GRAVY, molecular weight and cellular localization

\begin{tabular}{|c|c|c|c|c|c|c|c|c|c|c|c|c|}
\hline \multicolumn{13}{|l|}{ RICE } \\
\hline \multirow[t]{2}{*}{ MYB groups } & \multirow[t]{2}{*}{ No of genes } & \multirow[t]{2}{*}{ (\%) } & \multicolumn{3}{|c|}{ GRAVY } & \multicolumn{3}{|c|}{ PI } & \multicolumn{3}{|c|}{ Molecular weight } & \multirow[t]{2}{*}{ Localization } \\
\hline & & & Min. & Max. & Avg. & Min. & Max. & Avg. & Min. & Max. & Avg. & \\
\hline MYB-related genes & 62 & 40 & -1.287 & -0.201 & -1.3875 & 3.99 & 12.26 & 8.125 & 7613.7 & 170921.8 & 89267.75 & Nuclear \\
\hline MYB-R2R3 & 88 & 56.77 & -0.906 & -0.178 & -0.995 & 4.67 & 10.4 & 7.535 & 21605.3 & 75878.9 & 48742.1 & Nuclear \\
\hline MYB-R1R2R3 & 4 & 2.58 & -0.691 & -0.593 & -0.9875 & 5.05 & 8.53 & 13.605 & 64100.1 & 109413.5 & 86756.8 & Nuclear \\
\hline Atypical MYB genes & 1 & 0.64 & -0.748 & -0.748 & -0.748 & 9.56 & 9.56 & 9.56 & 92424.6 & 92424.6 & 92424.6 & Nuclear \\
\hline \multicolumn{13}{|l|}{ ARABIDOPSIS } \\
\hline \multirow[t]{2}{*}{ MYB groups } & No of genes & (\%) & \multicolumn{3}{|c|}{ GRAVY } & \multicolumn{3}{|c|}{ PI } & \multicolumn{3}{|c|}{ Molecular weight } & Localization \\
\hline & & & Min. & Max. & Avg. & Min. & Max. & Avg. & Min. & Max. & Avg. & \\
\hline MYB-related genes & 52 & 26.39 & -1.359 & 0.612 & -0.3735 & 4.75 & 6.62 & 2.375 & 7570.9 & 50112 & 3785.45 & Nuclear \\
\hline MYB-R2R3 & 138 & 70.05 & -1.102 & -0.471 & -0.7865 & 4.16 & 10.24 & 7.2 & 27951.2 & 33239 & 13975.6 & Nuclear \\
\hline MYB-R1R2R3 & 5 & 2.54 & -0.941 & -0.774 & -0.8575 & 5.43 & 9.22 & 7.325 & 50032.2 & 158268.4 & 79134.2 & Nuclear \\
\hline Atypical MYB genes & 2 & 0.51 & -0.941 & -0.94 & -0.9405 & 5.67 & 6.37 & 3.185 & 95766.5 & 96084.3 & 95925.4 & Nuclear \\
\hline
\end{tabular}

(AT5G41020) and 0.612 (AT1G35516) in Arabidopsis, respectively. We also calculated average isoelectric point (pI) value. The mean pI values for MYB-1R, R2R3 and R1R2R3 protein families were 7.55, 6.90 and 7.25 in rice, and 7.55, 6.89 and 6.80 in Arabidopsis, respectively. The average molecular weight of MYB-1R, R2R3 and R1R2R3 protein families were $31.128,34.561$ and $72.52 \mathrm{kDa}$ in rice, and 34.186, 35.875 and $86.217 \mathrm{kDa}$ in Arabidopsis, respectively (Additional file 1: Table S1).

\section{Functional classification of $M Y B$ transcription factors}

MYB proteins perform wide diversity of functions in plants. The R2R3-MYB proteins are involved in plant specific processes, such as control of secondary metabolism or cellular morphogenesis [43-49]. Gene ontology (GO) analysis suggested that R2R3-MYB genes, namely AtMYB16, AtMYB35, AtMYB5/AtMYB80, and AtMYB91 may regulate cell, anther, trichome and leaf morphogenesis, respectively. Likewise, R2R3-type genes, namely OsMYB16, OsMYB88, OsMYB117, LOC_Os01g50110 and LOC_Os03g38210 may regulate morphogenesis in rice. In addition to R2R3-type $M Y B s$, two $M Y B$-related genes, LOC_Os01g43180 and LOC_Os09g23200 may also regulate morphogenesis in rice. R2R3-type AtMYB10 and AT2G47210, MYB-related AT3G09600, and R1R2R3type $A t M Y B 3 R 4$ genes were identified with $\mathrm{GO}$ function, such as $\mathrm{N}$-terminal protein myristoylation, histone $\mathrm{H} 3$ acetylation, and regulation of DNA endoreduplication, respectively. Previous studies have shown that genes encoding 3R-MYB proteins have regulatory role in cell cycle control $[28,50]$. We also found that AtMYB3R4 may be involved in cell cycle control (GO: 0007049). GO analysis of MYB proteins illustrated that $98.70 \%$ OsMYB and $98.47 \% A t M Y B$ were fully involved in transcription activation, while rest of the MYB proteins were classified in to other GO functions, such as kinase activity, protein binding, transcription repressor activity, etc. GO analysis categorized rice LOC_Os01g62660 as signal transducer (GO: 0004871) and transcription activator. The R2R3type $A t M Y B 4$ was classified into transcriptional repressor group. The AtMYB4 expression is down regulated by exposure to UV-B light, indicating that derepression of its target genes is an important mechanism for acclimation to UV-B in Arabidopsis [51,52]. In our study, AtMYB34; a R2R3-type MYB protein, has been found with catalytickinase as well as transcription activator molecular functions as reported earlier [53,54]. The AtMYB34 is also involved in defense response against insects [55]. In consistent with previous report [56], AtMYB23 was found to have protein binding (i.e. interaction with GL3) as well as DNA-binding functions.

The subcellular localization of MYB proteins was predicted using several localization predictor softwares. The predicted locations of the MYB proteins were also verified by gene ontology under keyword "GO cellular component" and species-specific localization prediction tools, e.g., AtSubP for Arabidopsis [57] to enhance the accuracy of prediction. Consensus outcome revealed that $98.71 \%$ OsMYB and all AtMYB proteins were found to be nuclear localized and confirmed by the presence of nuclear localization signal (NLS). The remaining two members of MYB proteins in rice were predicted to be localized in mitochondria and plasma membrane. A Complete list of functional assignment of $M Y B$ genes is given in Additional file 2: Table S2.

\section{Gene structure and intron distribution}

To understand the structural components of $M Y B$ genes, their exon and intron organization was analyzed. We observed that 17 (10.96\%) OsMYB and 9 (4.56\%) 


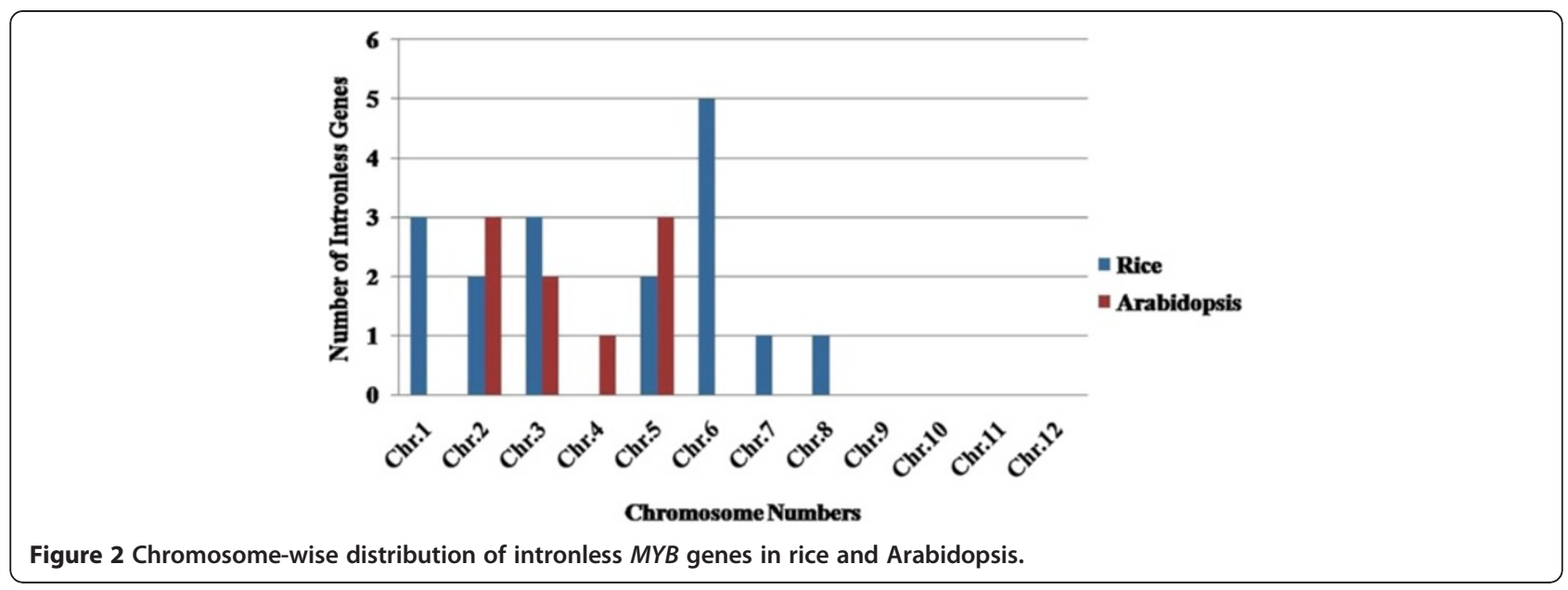

$A t M Y B$ genes were intronless (Figure 2), which is in conformity with the previous analysis [58]. To identify conserved intronless $M Y B$ genes, blastall (BLASTP) was performed between protein sequence of all the predicted intronless genes of rice and Arabidopsis, and vice versa. Expected cut-off value of $1 \mathrm{e}-6$ or less was used to identify the conserved intronless genes. We found that 13 (76.47\%) and 7 (77.77\%) intronless OsMYB and AtMYB genes, respectively, were orthologs. Other intronless $M Y B$ genes that fulfilled the matching criteria, expected cut-off value of $1 \mathrm{e}-10$ or less were referred to as paralogs. We observed that $4(23.52 \%)$ and $2(22.22 \%)$ intronless $O s M Y B$ and $A t M Y B$ genes, respectively, were paralogs (Additional file 3: Table S3). This analysis showed that intronless genes of rice and Arabidopsis are highly conserved, and may be involved in similar regulatory functions in these plants [36,58]. To explore the intron density in MYB genes with introns, we divided ORF into three zones, namely $\mathrm{N}$-terminal, central and $\mathrm{C}$-terminal zones. We observed that mid region had high density of introns, i.e., 43.99 and $50.63 \%$ in rice and Arabidopsis, respectively. The number of introns per ORF varied, with maximum of 12 and 15 introns in OsMYB4R1 and AT2G47210, respectively. Rice LOC_Os01g43180 and Arabidopsis AT3G10585 genes contain shortest introns with 37 and 43nt, respectively. Among all MYB genes, LOC_Os08g25799 of rice and AT1G35515 of Arabidopsis contained longest intron with an intron length of 5116 and 1621nt, respectively (Additional file 4: Table S4). In order to gain insight into exon-intron architecture, the intron positions on MYB domains were investigated. In support with previous results $[16,59]$, we also noticed that a large number of rice $(26.45 \%)$ and Arabidopsis (38.57\%) R2R3-type domain containing proteins have a conserved splicing pattern with three exons and two introns. However, some R2R3-type MYB genes lack one intron either in R2 or R3 repeat in rice (23.22\%) and
Arabidopsis (25.88\%) (Figure 3). It has been proposed that the duplication of R2 in an early form of two repeat MYB proteins gave rise to the R1R2R3 MYB domains [17]. Hence, we also investigated the exon-intron structure of R1R2R3-type MYB proteins. We observed that 3R-MYB proteins contained conserved three exons-two introns pattern in R1 and R2 and one conserved intron in R3 repeat in Arabidopsis. Similarly, in rice, three out of five 3R-MYB genes have similar structure (Figure 4; Additional file 4: Table S4). These results indicate similar distribution of introns in MYB domain in both rice and Arabidopsis.

\section{Chromosomal distribution, tandem repeats and duplication}

The position of all 155 OsMYB and 197 AtMYB genes were mapped on chromosome pseudomolecules available at MSU (release 5) for rice and TAIR (release 8) for Arabidopsis (Figures 5 and 6). The distribution and density of the $M Y B$ genes on chromosomes were not uniform. Some chromosomes and chromosomal regions have high density of the $M Y B$ genes than other regions. Rice chromosome 1 and Arabidopsis chromosome 5 contained highest density of $M Y B$ genes, i.e. 21.93 and $28.93 \%$, respectively. Conversely, chromosome 11 of rice and chromosome 2 of Arabidopsis contained lowest density of $M Y B$ genes, i.e. 2.58 and $12.69 \%$, respectively. Distribution of $M Y B$ genes on chromosomes revealed that lower arm of chromosomes are rich in $M Y B$ genes, i.e. $65.16 \%$ in rice and $52.79 \%$ in Arabidopsis. Distribution pattern also revealed that chromosome 5 in rice, and chromosome 2 and 5 in Arabidopsis contained higher number of $M Y B$ genes with introns, i.e. 29.41 and $33.33 \%$, respectively. Intronless $M Y B$ genes are absent in chromosome 4, 9, 10, 11 and 12 in rice, and chromosome 1 in Arabidopsis (Figure 2). Distribution of $M Y B$ genes on chromosomal loci revealed that 11 (7.09\%) 

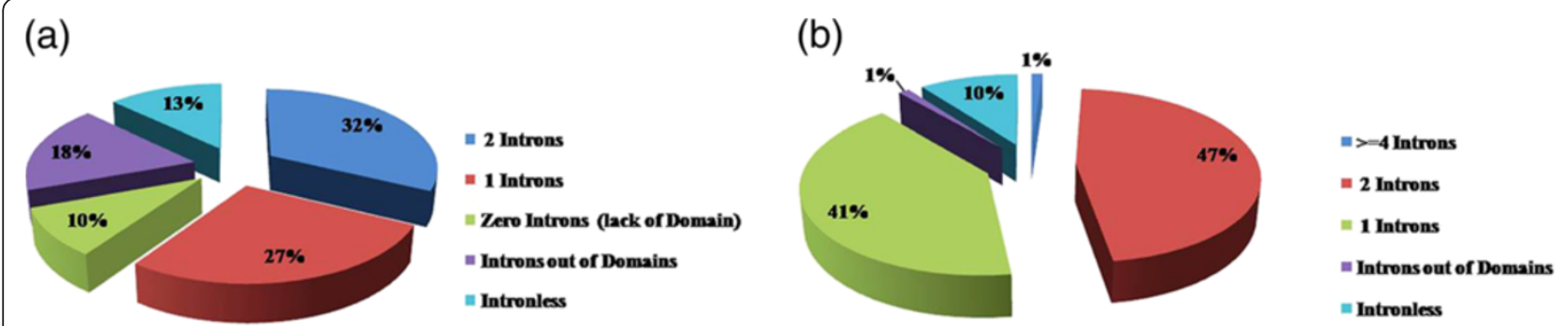

(c)
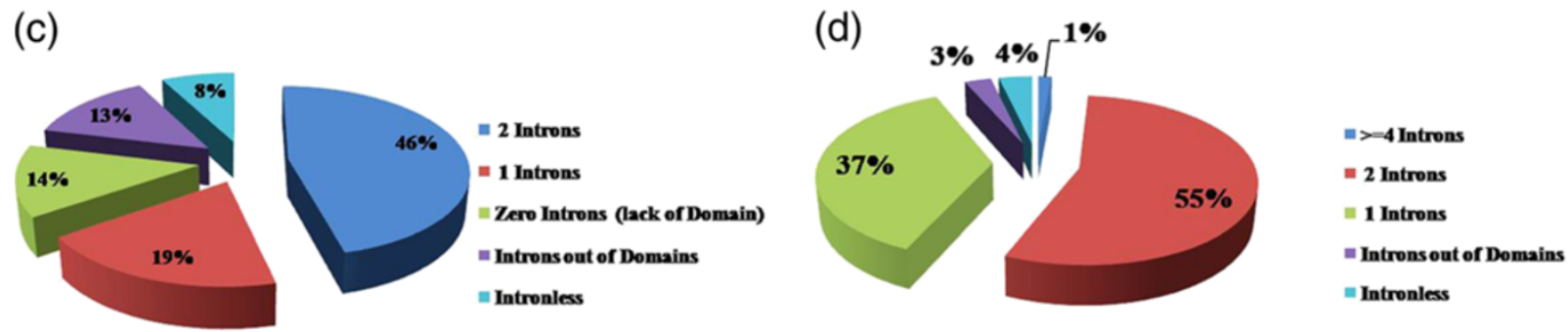

Figure 3 Intron distribution within the MYB domains of $M Y B$ genes in rice and Arabidopsis. The graph shows dominantly two intron positions within the domain of MYB-related $(\mathbf{a}, \mathbf{c})$ and R2R3-MYB genes $(\mathbf{b}, \mathbf{d})$ in rice and Arabidopsis, respectively.

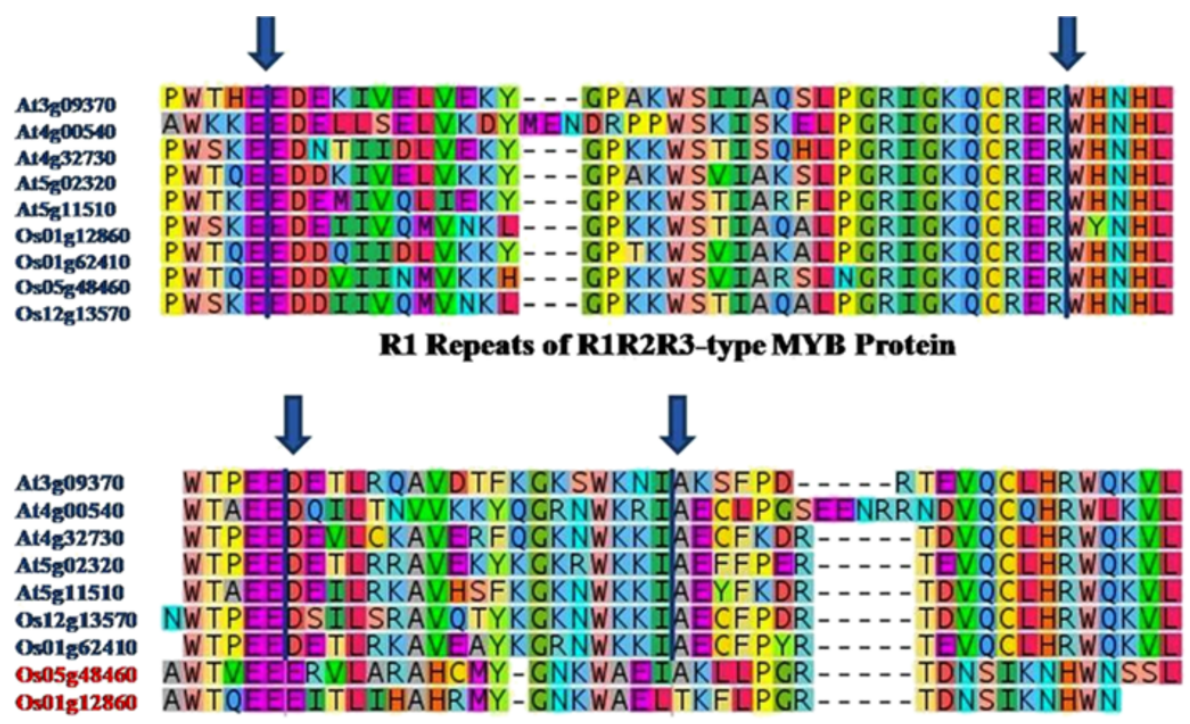

R2 Repeats of R1R2R3-type MYB Protein

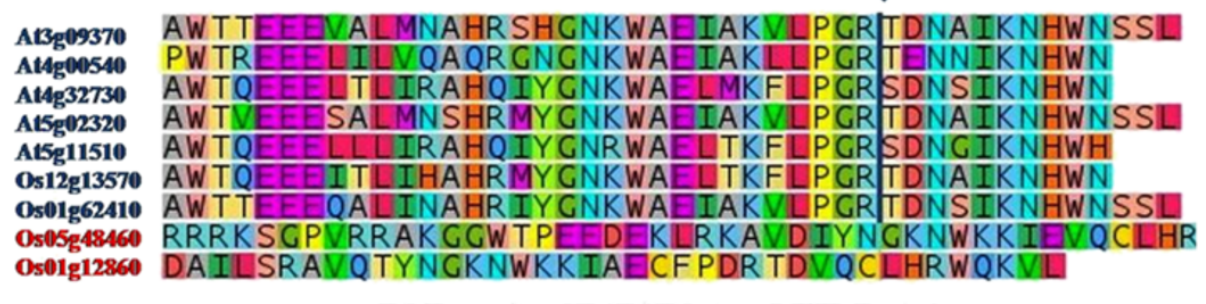

\section{R3 Repeats of R1R2R3-type MYB Protein}

Figure 4 Conserved intron position within the MYB domain of R1R2R3-type MYB genes in rice and Arabidopsis. Vertical bar and arrow indicate conserved introns position. MSU Gene IDs in red letters represent genes with non-conserved intron position. 


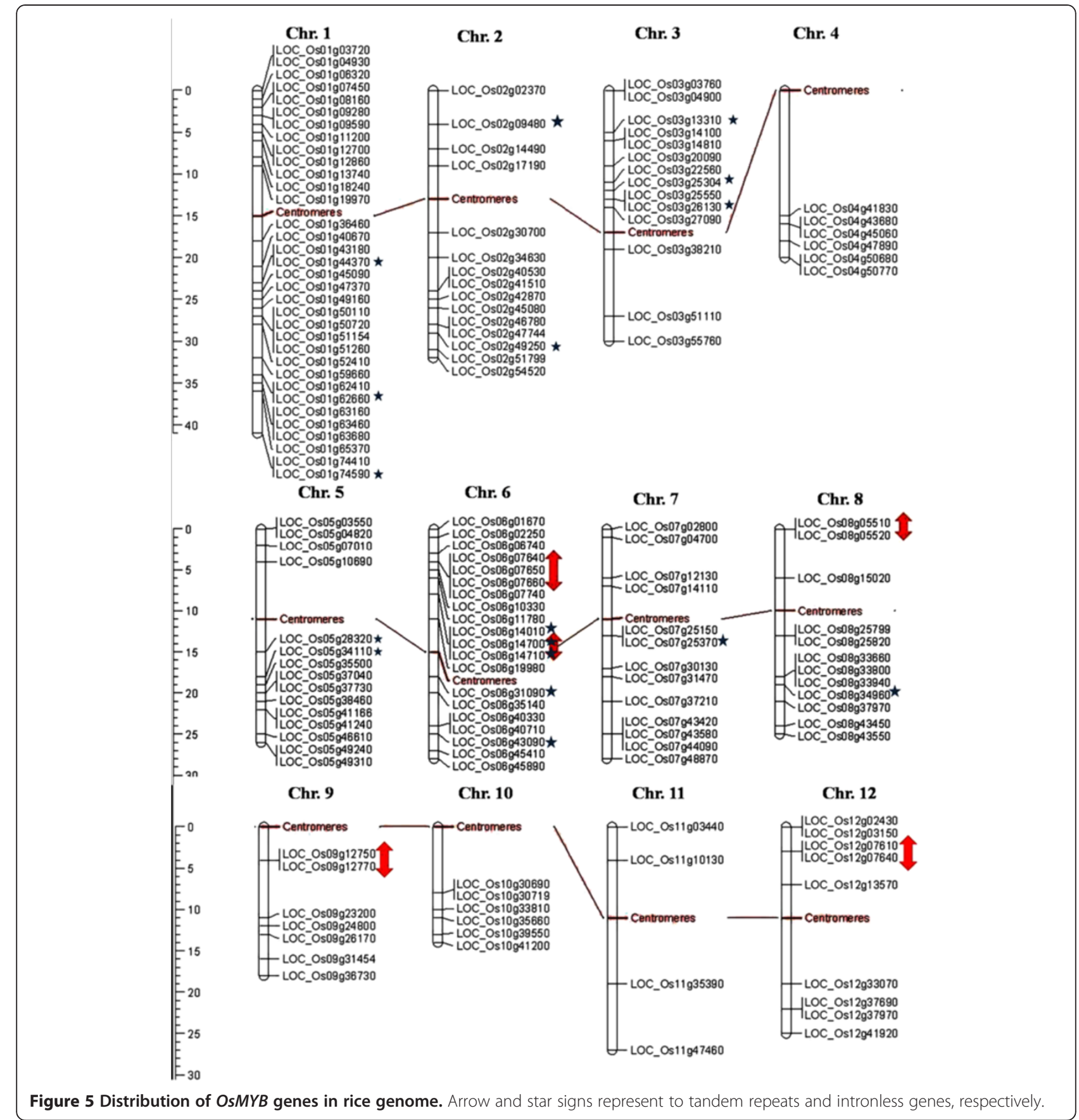

in rice and $20(10.15 \%)$ genes in Arabidopsis were found in tandem repeats suggesting local duplication (Table 2). Chromosome 6 in rice and chromosome 1 in Arabidopsis contained higher number of tandem repeats, i.e. 7 genes and showed over-representation of $M Y B$ genes. Three direct tandem repeats were found on chromosome 6 (LOC_Os06g07640; LOC_Os06g07650; LOC_Os06g07660) in rice, and chromosome 1 (AT1G66370, AT1G66380; AT1G66390) as well as chromosome 5 (AT5G40330; AT5G40350; AT5G40360) in Arabidopsis.
Four direct tandem repeats were also observed on chromosome 3 (AT3G10580, AT3G10585, AT3G10590 and AT3G10595) in Arabidopsis. Manual inspection unraveled $44(28.38 \%)$ and $69(35.02 \%)$ homologous pairs of $M Y B$ genes in rice and Arabidopsis, respectively evolved due to segmental duplication. We also observed that two homologous pairs in Arabidopsis contained one $M Y B$ gene and other than that was not classified as $M Y B$ gene in TAIR (release 10) databases (Table 3). About 44 (28.39\%) OsMYB and 69 (35.02\%) AtMYB genes 


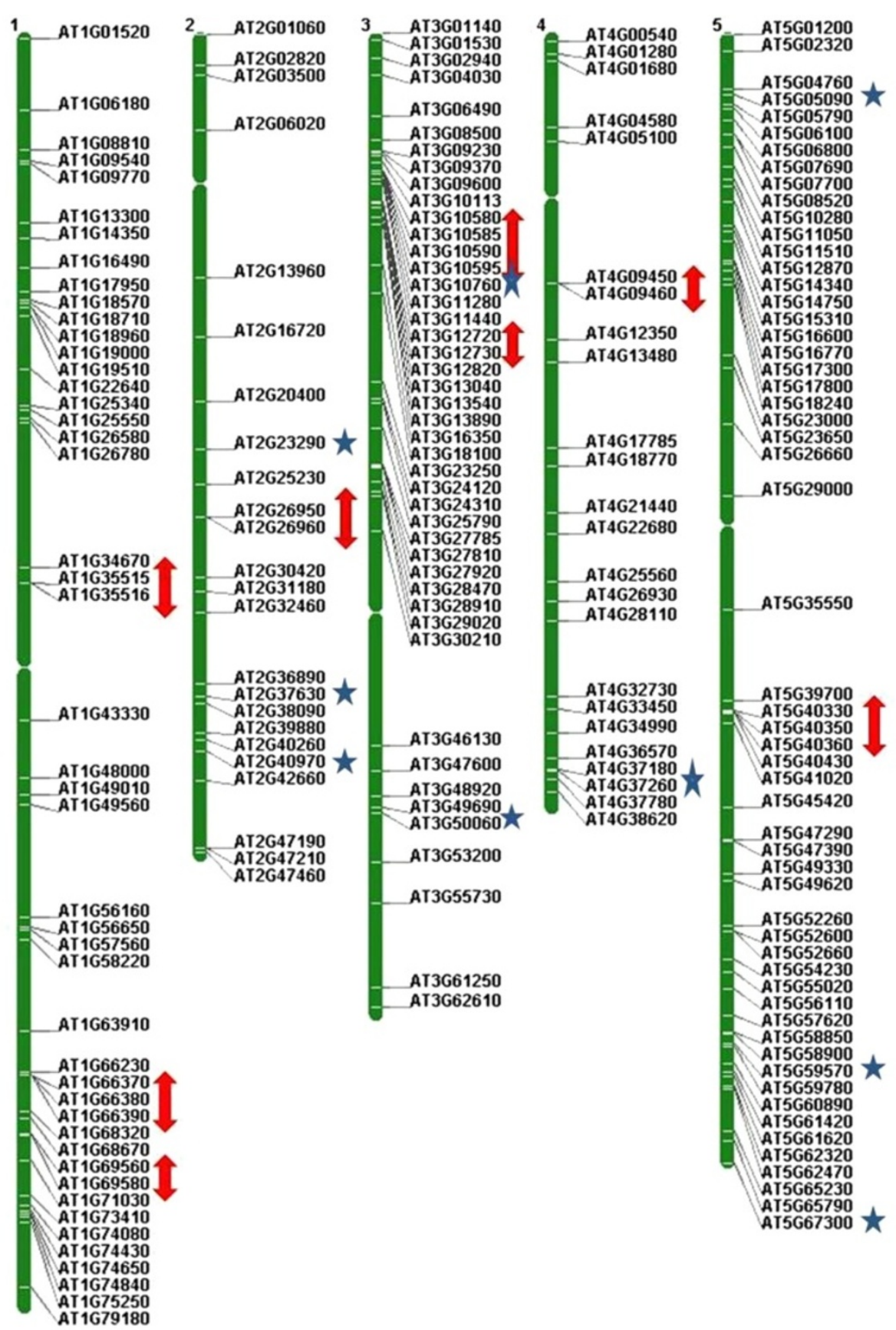

Figure 6 Distribution of AtMYB genes in Arabidopsis genome. Arrow and star signs represent tandem repeats and intronless genes, respectively.

showed homology with multiple genes including $M Y B$ genes from various locations on different chromosomes. It is widely accepted that redundant duplicated genes will be lost from the genome due to random mutation and loss of function, except when neo-or sub-functionalization occur [60,61]. Rabinowicz et al. (1999) suggested that gene duplications in R2R3-type $M Y B$ family occurred during earlier period of evolution in land plants [62]. Recently, a range of duplicated pair of $M Y B$ genes in R2R3-type protein family has been identified in maize [63]. Among the tandem repeat pair (AT2G26950 and AT2G26960) in Arabidopsis, AtMYB104 (AT2G26950) is down-regulated by $A B A$, anoxia and cold stress, but up-regulated under drought, high temperature and salt, while AtMYB81 (AT2G26960) expression pattern was opposite to that of AtMYB104, i.e., AtMYB81 is up-regulated in response to 
Table 2 Comparison of tandem repeat MYB genes in rice and Arabidopsis based on cellular localization

\begin{tabular}{|c|c|c|c|c|c|c|c|c|c|}
\hline \multirow[b]{2}{*}{ TR_NO } & \multicolumn{2}{|c|}{ Tandem repeat in rice } & \multirow[b]{2}{*}{ OsMYB_G1 } & \multirow[b]{2}{*}{ OsMYB_G2 } & \multirow[b]{2}{*}{$\begin{array}{l}\text { Cellular } \\
\text { localization } \\
\text { G1 }\end{array}$} & \multirow[b]{2}{*}{$\begin{array}{l}\text { Cellular } \\
\text { localization } \\
\text { G2 }\end{array}$} & \multicolumn{3}{|c|}{ Blast 2 sequences alignment } \\
\hline & TR_OsMYB_G1 & TR_OsMYB_G2 & & & & & $\begin{array}{l}\text { Bit } \\
\text { score }\end{array}$ & $\begin{array}{l}\% \\
\text { identity }\end{array}$ & E-value \\
\hline \multirow[t]{2}{*}{ OsTR1 } & LOC_Os06g07640 & LOC_Os06g07650 & OsMYB & OsMYB & Nuclear & Nuclear & 75.5 & $55 \%$ & $2.00 \mathrm{E}-18$ \\
\hline & LOC_Os06g07650 & LOC_Os06g07660 & OsMYB & OsMYB & Nuclear & Nuclear & 488 & $84 \%$ & $2.00 \mathrm{E}-142$ \\
\hline OsTR2 & LOC_Os06g14700 & LOC_Os06g 14710 & OsMYB & OsMYB & Nuclear & Nuclear & 146 & $64 \%$ & $2.00 \mathrm{E}-40$ \\
\hline OsTR3 & LOC_Os08g05510 & LOC_Os08g05520 & OsMYB & OsMYB103 & Nuclear & Nuclear & 19.2 & $25 \%$ & $1.60 \mathrm{E}-01$ \\
\hline OsTR4 & LOC_Os09g12750 & LOC_Os09g12770 & OsMYB & OsMYB & Nuclear & Nuclear & 55.8 & $40 \%$ & $6.00 \mathrm{E}-13$ \\
\hline \multirow[t]{2}{*}{ OsTR5 } & LOC_Os12g07610 & LOC_Os12g07640 & OsMYB & OsMYB & Nuclear & Nuclear & 105 & $45 \%$ & $2.00 \mathrm{E}-27$ \\
\hline & \multicolumn{2}{|c|}{ Tandem repeat in Arabidopsis } & & & & & \multicolumn{3}{|c|}{ Blast 2 sequences alignment } \\
\hline TR_NO & TR_AtMYB_G1 & TR_AtMYB_G2 & AtMYB_G1 & AtMYB_G2 & $\begin{array}{l}\text { Cellular } \\
\text { localization } \\
\text { G } 1\end{array}$ & $\begin{array}{l}\text { Cellular } \\
\text { localization } \\
\text { G2 }\end{array}$ & $\begin{array}{l}\text { Bit } \\
\text { score }\end{array}$ & $\begin{array}{l}\% \\
\text { identity }\end{array}$ & E-value \\
\hline AtTR1 & AT1G35515 & AT1G35516 & AtMYB8 & AtMYB & Nuclear & Nuclear & \multicolumn{3}{|c|}{ No significant similarity found } \\
\hline \multirow[t]{2}{*}{ AtTR2 } & AT1G66370 & AT1G66380 & AtMYB113 & AtMYB114 & Nuclear & Nuclear & 212 & $80 \%$ & $3.00 \mathrm{E}-60$ \\
\hline & AT1G66380 & AT1G66390 & AtMYB114 & AtMYB90 & Nuclear & Nuclear & 220 & $87 \%$ & $1.00 \mathrm{E}-62$ \\
\hline AtTR3 & AT1G69560 & AT1G69580 & AtMYB105 & AtMYB & Nuclear & Nuclear & 14.2 & $31 \%$ & 5.3 \\
\hline AtTR4 & AT2G26950 & AT2G26960 & AtMYB104 & AtMYB81 & Nuclear & Nuclear & 358 & $50 \%$ & 2.00E-103 \\
\hline \multirow[t]{2}{*}{ AtTR5 } & AT3G10580 & AT3G10585 & AtMYB & AtMYB & Nuclear & Nuclear & 172 & $64 \%$ & $4.00 \mathrm{E}-48$ \\
\hline & AT3G10590 & AT3G10595 & AtMYB & AtMYB & Nuclear & Nuclear & 56.6 & $27 \%$ & 3.00E-13 \\
\hline AtTR6 & AT3G12720 & AT3G12730 & AtMYB67 & AtMYB & Nuclear & Nuclear & 16.9 & $31 \%$ & 4.40E-01 \\
\hline AtTR7 & AT4G09450 & AT4G09460 & AtMYB & AtMYB6 & Cytoplasmic & Nuclear & 21.2 & $25 \%$ & 1.40E-02 \\
\hline \multirow[t]{2}{*}{ AtTR8 } & AT5G40330 & AT5G40350 & AtMYB23 & AtMYB24 & Nuclear & Nuclear & 142 & $55 \%$ & $5.00 \mathrm{E}-39$ \\
\hline & AT5G40350 & AT5G40360 & AtMYB24 & AtMYB115 & Nuclear & Nuclear & 89.4 & $42 \%$ & 8.00E-23 \\
\hline
\end{tabular}

MYB coding sequence were aligned using BLAST 2 SEQUENCES to quantitate the sequence differences between the paired genes.

ABA, anoxia and cold stress, but down regulated under drought, high temperature and salt stresses. Similar diversification was also observed in the duplicate pair (LOC_Os10g33810 and LOC_Os02g41510) in rice. OsMYB15 (LOC_Os10g33810) expressed in leaf, while LOC_Os02g41510 expressed in shoot and panicle tissue. These spatial and temporal differences among different $M Y B$ genes evolved by duplication indicate their functional diversification.

\section{Cis-motifs in the MYB gene promoters}

Discovery of regulatory cis-elements in the promoter regions is essential to understand the spatial and temporal expression pattern of $M Y B$ genes. Co-expressed genes may be regulated by a common set of transcription factors, and can be detected by the occurrence of specific cis-regulatory motifs in the promoter region. Hence, we analyzed the promoter regions of the drought up- and down-regulated $M Y B$ genes identified from our previous microarray data experiments [64]. Among the top five cis-motifs identified by this analysis, only CCA1 (TTWKTTWWTTTT) was the previously known cismotif. Although, CCA1 cis-motif was reported as common feature of rice genome [65], we found CCA1 cis-motif only in genes that are down-regulated by drought stress (Figure 7). The CCA1 motif was found in $94.74 \%$ of the drought down-regulated genes in rice. Furthermore, we investigated the group of R2R3-type $M Y B$ genes for the discovery of gene-specific new cis-regulatory element in both rice and Arabidopsis. Likewise, we discovered novel cis-motifs with no description in PLACE database, except for CCA1 motif in rice (Figure 7). The CCA1 motif was found in $70.45 \%$ of the R2R3-type $M Y B$ genes in rice. The CCA1, a MYB-related TF, binds to CCA1 motif and regulate circadian clock controlled expression of genes in Arabidopsis [66]. To validate our prediction, we examined the diurnal or circadian clock controlled MYB expression using "Diurnal Version 2.0" [67]. About 47.74 and 90.86\% $M Y B$ genes were found to be diurnal/circadian-regulated in rice and Arabidopsis, respectively (Additional file 5: Table S5). Noticeably, we did not find any common motif between rice and Arabidopsis $M Y B$ promoter regions, indicating divergence in regulatory region of $M Y B$ genes between monocot and dicot species.

\section{Expression of $M Y B$ genes under abiotic stresses}

To identify $M Y B$ genes with a potential role in abiotic stress response of plants, we analyzed the expression 
Table 3 Comparison of homologous pair of MYB genes of rice and Arabidopsis based on cellular localization

\begin{tabular}{|c|c|c|c|c|c|c|c|c|c|}
\hline \multirow[b]{2}{*}{ HP_NO } & \multicolumn{2}{|c|}{ Duplications in rice } & \multirow[b]{2}{*}{ OsMYB_G1 } & \multirow[b]{2}{*}{ OsMYB_G2 } & \multirow[b]{2}{*}{$\begin{array}{l}\text { Cellular } \\
\text { localization } \\
\text { G } 1\end{array}$} & \multirow[b]{2}{*}{$\begin{array}{l}\text { Cellular } \\
\text { localization } \\
\text { G2 }\end{array}$} & \multicolumn{3}{|c|}{ Blast 2 sequences alignment } \\
\hline & OsMYB_HP_G1 & OsMYB_HP_G2 & & & & & $\begin{array}{l}\text { Bit } \\
\text { score }\end{array}$ & $\begin{array}{l}\% \\
\text { identity }\end{array}$ & E-value \\
\hline OsHP1 & LOC_Os01g06320 & LOC_Os05g07010 & OsMYB & OsMYB & Nuclear & Nuclear & 160 & $81 \%$ & $1.00 \mathrm{E}-38$ \\
\hline OsHP2 & LOC_Os01g18240 & LOC_Os05g04820 & OsMYB & OsMYB & Nuclear & Nuclear & 1230 & $79 \%$ & $0.00 \mathrm{E}+00$ \\
\hline OsHP3 & LOC_Os01g44370 & LOC_Os05g50350 & OsMYB & OsMYB & Nuclear & Nuclear & 234 & $82 \%$ & $8.00 \mathrm{E}-59$ \\
\hline OsHP4 & LOC_Os01g47370 & LOC_Os05g49240 & OsMYB & OsMYB & Nuclear & Nuclear & 188 & $77 \%$ & $3.00 \mathrm{E}-47$ \\
\hline OsHP5 & LOC_Os01g49160 & LOC_Os05g48010 & OsMYB & OsMYB & Nuclear & Nuclear & 234 & $94 \%$ & $2.00 \mathrm{E}-58$ \\
\hline OsHP6 & LOC_Os01g50720 & LOC_Os05g46610 & OsMYB & OsMYB & Nuclear & Nuclear & 696 & $77 \%$ & $0.00 \mathrm{E}+00$ \\
\hline OsHP7 & LOC_Os01g59660 & LOC_Os05g41166 & GAMYB & OsMYB & Nuclear & Nuclear & 298 & $78 \%$ & $1.00 \mathrm{E}-75$ \\
\hline OsHP8 & LOC_Os01g62410 & LOC_Os05g38460 & OsMYB3R-2 & OsMYB & Nuclear & Nuclear & 476 & $74 \%$ & $8.00 \mathrm{E}-124$ \\
\hline OsHP9 & LOC_Os01g63460 & LOC_Os05g37730 & OsMYB & OsMYB & Nuclear & Nuclear & 22 & $100 \%$ & $6.80 \mathrm{E}-01$ \\
\hline OsHP10 & LOC_Os01g65370 & LOC_Os05g35500 & OsMYB3 & OsMYB & Nuclear & Nuclear & 636 & $88 \%$ & $6.00 \mathrm{E}-168$ \\
\hline OsHP11 & LOC_Os02g09480 & LOC_Os05g37730 & OsMYB & OsMYB & Nuclear & Nuclear & 32 & $87 \%$ & 7.00E-04 \\
\hline OsHP12 & LOC_Os02g14490 & LOC_Os06g35140 & OsMYB & OsMYB & Nuclear & Nuclear & 548 & $73 \%$ & $2.00 \mathrm{E}-143$ \\
\hline OsHP13 & LOC_Os02g40530 & LOC_Os04g42950 & OsMYB & OsMYB & Nuclear & Nuclear & 284 & $94 \%$ & $8.00 \mathrm{E}-72$ \\
\hline OsHP14 & LOC_Os02g41510 & LOC_Os04g43680 & OsMYB & OsMYB4 & Nuclear & Nuclear & 460 & $86 \%$ & $3.00 \mathrm{E}-120$ \\
\hline OsHP15 & LOC_Os02g42870 & LOC_Os04g45060 & OsMYB & OsMYB & Nuclear & Nuclear & 744 & $77 \%$ & $0.00 \mathrm{E}+00$ \\
\hline OsHP16 & LOC_Os02g45080 & LOC_Os04g47890 & OsMYB & OsMYB & Nuclear & Nuclear & 312 & $73 \%$ & $6.00 \mathrm{E}-80$ \\
\hline OsHP17 & LOC_Os02g46780 & LOC_Os04g50770 & OsMYB & OsMYB & Nuclear & Nuclear & 620 & $70 \%$ & $2.00 \mathrm{E}-163$ \\
\hline OsHP18 & LOC_Os02g51799 & LOC_Os06g11780 & OsMYB & OsMYB93 & Nuclear & Nuclear & 442 & $80 \%$ & $5.00 \mathrm{E}-115$ \\
\hline OsHP19 & LOC_Os02g54520 & LOC_Os07g48870 & OsMYB & OsMYB2 & Nuclear & Nuclear & 54 & $78 \%$ & $1.00 \mathrm{E}-09$ \\
\hline OsHP20 & LOC_Os03g03760 & LOC_Os10g39550 & OsMYB & OsMYB & Nuclear & Nuclear & 136 & $83 \%$ & $3.00 \mathrm{E}-31$ \\
\hline OsHP21 & LOC_Os03g20090 & LOC_Os07g48870 & OsMYB112 & OsMYB2 & Nuclear & Nuclear & 554 & $84 \%$ & $2.00 \mathrm{E}-145$ \\
\hline OsHP22 & LOC_Os03g25550 & LOC_Os07g44090 & OsMYB & OsMYB & Nuclear & Nuclear & 374 & $88 \%$ & $1.00 \mathrm{E}-96$ \\
\hline OsHP23 & LOC_Os03g26130 & LOC_Os07g43580 & OsMYB & OsMYB30 & Nuclear & Nuclear & 384 & $82 \%$ & 2.00E-99 \\
\hline OsHP24 & LOC_Os05g04820 & LOC_Os07g44090 & OsMYB & OsMYB & Nuclear & Nuclear & 422 & $83 \%$ & 2.00E-109 \\
\hline OsHP25 & LOC_Os05g10690 & LOC_Os01g09640 & OsMYB & OsMYB & Nuclear & Nuclear & 232 & $83 \%$ & $9.00 \mathrm{E}-58$ \\
\hline OsHP26 & LOC_Os05g49240 & LOC_Os05g50340 & OsMYB & OsMYB & Nuclear & Nuclear & 104 & $72 \%$ & 4.00E-24 \\
\hline OsHP27 & LOC_Os06g43090 & LOC_Os02g09480 & OsMYB & OsMYB & Nuclear & Nuclear & 616 & $71 \%$ & $2.00 \mathrm{E}-162$ \\
\hline OsHP28 & LOC_Os06g45410 & LOC_Os02g07770 & OsMYB & OsMYB & Nuclear & Nuclear & 180 & $90 \%$ & $1.00 \mathrm{E}-43$ \\
\hline OsHP29 & LOC_Os06g45890 & LOC_Os02g07170 & OsMYB & OsMYB & Nuclear & Nuclear & 98 & $81 \%$ & $1.00 \mathrm{E}-21$ \\
\hline OsHP30 & LOC_Os07g02800 & LOC_Os03g55590 & OsMYB & OsMYB & Nuclear & Nuclear & 162 & $91 \%$ & $1.00 \mathrm{E}-38$ \\
\hline OsHP31 & LOC_Os08g25799 & LOC_Os09g12750 & OsMYB & OsMYB & Nuclear & Nuclear & 682 & $80 \%$ & $2.00 \mathrm{E}-180$ \\
\hline OsHP32 & LOC_Os08g25820 & LOC_Os09g12770 & OsMYB & OsMYB & Nuclear & Nuclear & 616 & $73 \%$ & $2.00 \mathrm{E}-162$ \\
\hline OsHP33 & LOC_Os08g33660 & LOC_Os02g36890 & OsMYB16 & OsMYB & Nuclear & Nuclear & 134 & $69 \%$ & 4.00E-31 \\
\hline OsHP34 & LOC_Os08g33660 & LOC_Os04g38740 & OsMYB16 & OsMYB & Nuclear & Nuclear & 136 & $80 \%$ & $1.00 \mathrm{E}-31$ \\
\hline OsHP35 & LOC_Os08g33940 & LOC_Os09g24800 & OsMYB & OsMYB & Nuclear & Nuclear & 838 & $76 \%$ & $0.00 \mathrm{E}+00$ \\
\hline OsHP36 & LOC_Os08g43450 & LOC_Os09g36250 & OsMYB & OsMYB & Nuclear & Nuclear & 76 & $71 \%$ & $2.00 \mathrm{E}-15$ \\
\hline OsHP37 & LOC_Os08g43550 & LOC_Os09g36730 & OsMYB7 & OsMYB & Nuclear & Nuclear & 502 & $84 \%$ & $1.00 \mathrm{E}-131$ \\
\hline OsHP38 & LOC_Os09g23200 & LOC_Os08g33050 & OsMYB & OsMYB & Nuclear & Nuclear & 222 & $66 \%$ & $2.00 \mathrm{E}-54$ \\
\hline OsHP39 & LOC_Os10g33810 & LOC_Os02g41510 & OsMYB15 & OsMYB & Nuclear & Nuclear & 374 & $81 \%$ & 8.00E-97 \\
\hline OsHP40 & LOC_Os10g33810 & LOC_Os04g43680 & OsMYB15 & OsMYB4 & Nuclear & Nuclear & 384 & $82 \%$ & 2.00E-99 \\
\hline OsHP41 & LOC_Os10g39550 & LOC_Os03g03760 & OsMYB & OsMYB & Nuclear & Nuclear & 384 & $81 \%$ & 3.00E-99 \\
\hline OsHP42 & LOC_Os11g03440 & LOC_Os12g03150 & OsMYB & OsMYB & Nuclear & Nuclear & 1702 & $96 \%$ & $0.00 \mathrm{E}+00$ \\
\hline OsHP43 & LOC_Os11g47460 & LOC_Os12g37970 & OsMYB & OsMYB & Nuclear & Nuclear & 634 & $83 \%$ & $2.00 \mathrm{E}-167$ \\
\hline OsHP44 & LOC_Os12g37690 & LOC_Os11g45740 & OsMYB78 & OsMYB & Nuclear & Nuclear & 226 & $88 \%$ & $5.00 \mathrm{E}-56$ \\
\hline
\end{tabular}


Table 3 Comparison of homologous pair of MYB genes of rice and Arabidopsis based on cellular localization (Continued)

\begin{tabular}{|c|c|c|c|c|c|c|c|c|c|}
\hline \multirow[b]{2}{*}{ HP_NO } & \multicolumn{2}{|c|}{ Duplications in Arabidopsis } & \multirow[b]{2}{*}{ AtMYB_G1 } & \multirow[b]{2}{*}{ ATMYB_G2 } & \multirow[b]{2}{*}{$\begin{array}{l}\text { Cellular } \\
\text { localization } \\
\text { G } 1\end{array}$} & \multirow[b]{2}{*}{$\begin{array}{l}\text { Cellular } \\
\text { localization } \\
\text { G2 }\end{array}$} & \multicolumn{3}{|c|}{ Blast 2 sequences alignment } \\
\hline & AtMYB_HP_G1 & AtMYB_HP_G2 & & & & & $\begin{array}{l}\text { Bit } \\
\text { score }\end{array}$ & $\begin{array}{l}\% \\
\text { identity }\end{array}$ & E-value \\
\hline AtHP1 & AT2G31180 & AT1G06180 & AtMYB14 & AtMYB13 & Nuclear & Nuclear & 350 & $84 \%$ & $2.00 \mathrm{E}-100$ \\
\hline AtHP2 & AT1G57560 & AT1G09540 & AtMYB50 & AtMYB61 & Nuclear & Nuclear & 392 & $88 \%$ & 7.00E-113 \\
\hline AtHP3 & AT1G58220 & AT1G09710 & AtMYB1I & AtMYB & Nuclear & Nuclear & 827 & $75 \%$ & 0 \\
\hline AtHP4 & AT1G26580 & AT1G13880 & AtMYB & No MYB & Nuclear & Nuclear & 45.4 & $76 \%$ & 4.00E-08 \\
\hline AtHP5 & AT2G02820 & AT1G14350 & AtMYB88 & AtMYB124 & Nuclear & Nuclear & 728 & $80 \%$ & 0 \\
\hline AtHP6 & AT3G12820 & AT1G16490 & AtMYB10 & AtMYB58 & Nuclear & Nuclear & 293 & $79 \%$ & $3.00 E-83$ \\
\hline AtHP7 & AT1G17950 & AT1G73410 & AtMYB52 & AtMYB54 & Nuclear & Nuclear & 381 & $88 \%$ & 7.00E-110 \\
\hline AtHP8 & AT1G79180 & AT1G16490 & AtMYB63 & AtMYB58 & Nuclear & Nuclear & 346 & $84 \%$ & 4.00E-99 \\
\hline AtHP9 & AT5G61420 & AT1G18570 & AtMYB28 & AtMYB51 & Nuclear & Nuclear & 99 & $86 \%$ & $1.00 \mathrm{E}-24$ \\
\hline AtHP10 & AT1G74080 & AT1G18570 & AtMYB122 & AtMYB51 & Nuclear & Nuclear & 305 & $81 \%$ & $9.00 \mathrm{E}-87$ \\
\hline AtHP11 & AT5G07700 & AT1G18570 & AtMYB76 & AtMYB51 & Nuclear & Nuclear & 185 & $71 \%$ & $2.00 \mathrm{E}-50$ \\
\hline AtHP12 & AT5G60890 & AT1G18570 & AtMYB34 & AtMYB51 & Nuclear & Nuclear & 206 & $77 \%$ & $8.00 \mathrm{E}-57$ \\
\hline AtHP13 & AT1G74430 & AT1G18710 & AtMYB95 & AtMYB47 & Nuclear & Nuclear & 351 & $82 \%$ & 7.00E-101 \\
\hline AtHP14 & AT1G74840 & AT1G19000 & AtMYB & AtMYB & Nuclear & Nuclear & 233 & $85 \%$ & $3.00 \mathrm{E}-65$ \\
\hline AtHP15 & AT1G35516 & AT1G22640 & AtMYB & AtMYB3 & Nuclear & Nuclear & $\begin{array}{l}\text { No significant } \\
\text { similarity found }\end{array}$ & & \\
\hline AtHP16 & AT4G09460 & AT1G22640 & AtMYB6 & AtMYB3 & Nuclear & Nuclear & 394 & $84 \%$ & 1.00E-113 \\
\hline AtHP17 & AT1G68320 & AT1G25340 & AtMYB62 & AtMYB116 & Nuclear & Nuclear & 366 & $86 \%$ & $3.00 \mathrm{E}-105$ \\
\hline AtHP18 & AT3G27810 & AT1G25340 & AtMYB21 & AtMYB116 & Nuclear & Nuclear & 149 & $70 \%$ & $7.00 \mathrm{E}-40$ \\
\hline AtHP19 & AT1G68670 & AT1G25550 & AtMYB & AtMYB & Nuclear & Nuclear & 176 & $84 \%$ & $8.00 \mathrm{E}-48$ \\
\hline AtHP20 & AT3G29020 & AT1G26780 & AtMYB110 & AtMYB117 & Nuclear & Nuclear & 232 & $77 \%$ & $8.00 \mathrm{E}-65$ \\
\hline AtHP21 & AT1G26780 & AT1G69560 & AtMYB117 & AtMYB105 & Nuclear & Nuclear & 416 & $88 \%$ & $3.00 \mathrm{E}-120$ \\
\hline AtHP22 & AT5G39700 & AT1G69560 & AtMYB89 & AtMYB105 & Nuclear & Nuclear & $\begin{array}{l}\text { No significant } \\
\text { similarity found }\end{array}$ & & \\
\hline AtHP23 & AT5G07690 & AT1G74080 & AtMYB29 & AtMYB122 & Nuclear & Nuclear & 161 & $76 \%$ & $2.00 \mathrm{E}-43$ \\
\hline AtHP24 & AT1G19510 & AT1G75250 & AtMYB & AtMYB & Nuclear & Nuclear & 154 & $80 \%$ & 4.00E-42 \\
\hline AtHP25 & AT4G36570 & AT1G75250 & AtMYB & AtMYB & Nuclear & Nuclear & $\begin{array}{l}\text { No significant } \\
\text { similarity found }\end{array}$ & & \\
\hline AtHP26 & AT4G34990 & AT2G16720 & AtMYB32 & AtMYB7 & Nuclear & Nuclear & 411 & $85 \%$ & 1.00E-118 \\
\hline AtHP27 & AT4G37260 & AT2G23290 & AtMYB73 & AtMYB70 & Nuclear & Nuclear & 364 & $84 \%$ & 1.00E-104 \\
\hline AtHP28 & AT5G67300 & AT2G23290 & AtMYB44 & AtMYB70 & Nuclear & Nuclear & 171 & $77 \%$ & $3.00 \mathrm{E}-46$ \\
\hline AtHP29 & AT5G1 1050 & AT2G25230 & AtMYB64 & AtMYB100 & Nuclear & Nuclear & 63.9 & $78 \%$ & $1.00 \mathrm{E}-13$ \\
\hline AtHP30 & AT5G01200 & AT2G38090 & AtMYB & AtMYB & Nuclear & Nuclear & 195 & $82 \%$ & $1.00 \mathrm{E}-53$ \\
\hline AtHP31 & AT3G55730 & AT2G39880 & AtMYB109 & AtMYB25 & Nuclear & Nuclear & 281 & $81 \%$ & $2.00 \mathrm{E}-79$ \\
\hline AtHP32 & AT3G10760 & AT2G40970 & AtMYB & AtMYB & Nuclear & Nuclear & 235 & $69 \%$ & $8.00 \mathrm{E}-66$ \\
\hline AtHP33 & AT5G05090 & AT2G40970 & AtMYB & AtMYB & Nuclear & Nuclear & 156 & $81 \%$ & $5.00 \mathrm{E}-42$ \\
\hline AtHP34 & AT3G62610 & AT2G47460 & AtMYB11 & AtMYB12 & Nuclear & Nuclear & 388 & $86 \%$ & $9.00 \mathrm{E}-112$ \\
\hline AtHP35 & AT5G15310 & AT3G01140 & AtMYB16 & AtMYB106 & Nuclear & Nuclear & 593 & $83 \%$ & $2.00 \mathrm{E}-173$ \\
\hline AtHP36 & AT5G40350 & AT3G01530 & AtMYB24 & AtMYB57 & Nuclear & Nuclear & 254 & $81 \%$ & 1.00E-71 \\
\hline AtHP37 & AT5G16600 & AT3G02940 & AtMYB43 & AtMYB107 & Nuclear & Nuclear & 110 & $73 \%$ & $7.00 \mathrm{E}-28$ \\
\hline AtHP38 & AT5G16770 & AT3G02940 & AtMYB9 & AtMYB107 & Nuclear & Nuclear & 586 & $86 \%$ & $3.00 \mathrm{E}-171$ \\
\hline AtHP39 & AT3G24120 & AT3G04030 & AtMYB3I & AtMYB & Nuclear & Nuclear & $73 \%$ & 86 & 1.00E-20 \\
\hline AtHP40 & AT5G18240 & AT3G04030 & AtMYB & AtMYB & Nuclear & Nuclear & 887 & $80 \%$ & 0 \\
\hline AtHP41 & AT5G49620 & AT3G06490 & AtMYB78 & AtMYB108 & Nuclear & Nuclear & 396 & $83 \%$ & 4.00E-114 \\
\hline
\end{tabular}


Table 3 Comparison of homologous pair of MYB genes of rice and Arabidopsis based on cellular localization (Continued)

\begin{tabular}{|c|c|c|c|c|c|c|c|c|c|}
\hline AtHP42 & AT5G02320 & AT3G09370 & AtMYB3R5 & AtMYB3R3 & Nuclear & Nuclear & 610 & $85 \%$ & $4.00 \mathrm{E}-178$ \\
\hline AtHP43 & AT5G04760 & AT3G10580 & AtMYB & AtMYB & Nuclear & Nuclear & 105 & $71 \%$ & $7.00 \mathrm{E}-27$ \\
\hline AtHP44 & AT5G05790 & AT3G11280 & AtMYB & AtMYB & Nuclear & Nuclear & 455 & $80 \%$ & $5.00 \mathrm{E}-132$ \\
\hline AtHP45 & AT5G06100 & AT3G11440 & AtMYB33 & AtMYB65 & Nuclear & Nuclear & 710 & $78 \%$ & 0 \\
\hline AtHP46 & AT1G56160 & AT3G12820 & AtMYB72 & AtMYB10 & Nuclear & Nuclear & 270 & $81 \%$ & $2.00 \mathrm{E}-76$ \\
\hline AtHP47 & AT4G13480 & AT3G24310 & AtMYB79 & AtMYB71 & Nuclear & Nuclear & 436 & $83 \%$ & $2.00 \mathrm{E}-126$ \\
\hline AtHP48 & AT1G13300 & AT3G25790 & AtMYB & AtMYB & Nuclear & Nuclear & 250 & $84 \%$ & 4.00E-70 \\
\hline AtHP49 & AT5G40360 & AT3G27785 & AtMYB115 & AtMYB118 & Nuclear & Nuclear & 161 & $76 \%$ & $3.00 \mathrm{E}-43$ \\
\hline AtHP50 & AT3G01530 & At1g68320 & AtMYB57 & AtMYB62 & Nuclear & Nuclear & 239 & $81 \%$ & 4.00E-67 \\
\hline AtHP51 & AT5G14750 & AT3G27920 & AtMYB66 & AtMYBO & Nuclear & Nuclear & 320 & $80 \%$ & 1.00E-91 \\
\hline AtHP52 & AT5G40330 & AT3G27920 & AtMYB23 & AtMYBO & Nuclear & Nuclear & 379 & $85 \%$ & 2.00E-109 \\
\hline AtHP53 & AT5G59780 & AT3G46130 & AtMYB59 & AtMYB48 & Nuclear & Nuclear & 237 & $86 \%$ & $1.00 \mathrm{E}-66$ \\
\hline AtHP54 & AT5G59570 & AT3G46640 & AtMYB & AtMYB & Nuclear & Nuclear & 313 & $85 \%$ & 4.00E-89 \\
\hline AtHP55 & AT5G62470 & AT3G47600 & AtMYB96 & AtMYB94 & Nuclear & Nuclear & 527 & $88 \%$ & 2.00E-153 \\
\hline AtHP56 & AT5G65790 & AT3G49690 & AtMYB68 & AtMYB84 & Nuclear & Nuclear & 494 & $87 \%$ & 2.00E-143 \\
\hline AtHP57 & AT4G37780 & AT3G49690 & AtMYB87 & AtMYB84 & Nuclear & Nuclear & 246 & $79 \%$ & 4.00E-69 \\
\hline AtHP58 & AT4G22680 & AT3G61250 & AtMYB85 & AtMYB17 & Nuclear & Nuclear & 147 & $70 \%$ & $3.00 E-39$ \\
\hline AtHP59 & AT1G01520 & AT4G01280 & AtMYB & AtMYB & Nuclear & Nuclear & 272 & $83 \%$ & 7.00E-77 \\
\hline AtHP60 & AT4G21440 & AT4G05100 & AtMYB102 & AtMYB74 & Nuclear & Nuclear & 385 & $89 \%$ & $1.00 \mathrm{E}-110$ \\
\hline AtHP61 & AT5G52260 & AT4G25560 & AtMYB19 & AtMYB18 & Nuclear & Nuclear & 407 & $79 \%$ & 2.00E-117 \\
\hline AtHP62 & AT5G55020 & AT4G26930 & AtMYB120 & AtMYB97 & Nuclear & Nuclear & 283 & $82 \%$ & $7.00 \mathrm{E}-80$ \\
\hline AtHP63 & AT2G20400 & AT4G28610 & AtMYB & No MYB & Nuclear & Nuclear & 419 & $73 \%$ & 7.00E-121 \\
\hline AtHP64 & AT5G11510 & AT4G32730 & AtMYB3R4 & AtMYB3R1 & Nuclear & Nuclear & 329 & $78 \%$ & $3.00 \mathrm{E}-93$ \\
\hline AtHP65 & AT3G09600 & AT5G02840 & AtMYB & MYB (LCL1) & Nuclear & Nuclear & 682 & $80 \%$ & 0 \\
\hline AtHP66 & AT3G10590 & AT5G04760 & AtMYB & AtMYB & Nuclear & Nuclear & 51.8 & $76 \%$ & $1.00 \mathrm{E}-10$ \\
\hline AtHP67 & AT5G23650 & AT5G08520 & AtMYB & AtMYB & Nuclear & Nuclear & 139 & $72 \%$ & $8.00 \mathrm{E}-37$ \\
\hline AtHP68 & AT5G65230 & AT5G10280 & AtMYB53 & AtMYB92 & Nuclear & Nuclear & 534 & $84 \%$ & 9.00E-156 \\
\hline AtHP69 & AT3G50060 & AT5G67300 & AtMYB77 & AtMYB44 & Nuclear & Nuclear & 265 & $82 \%$ & 1.00E-74 \\
\hline
\end{tabular}

The coding sequence were aligned using BLAST 2 SEQUENCES to quantitate the sequence differences between the gene pairs.

pattern of $M Y B$ genes in response to abiotic stresses. Expression of $M Y B s$ genes was examined from the availability of full-length cDNA (FL-CDNA) and Expressed Sequence Tag (EST) available at MSU and dbEST databases for rice and Arabidopsis, respectively [68]. It was found that 109 OsMYB genes in rice and 157 AtMYB genes in Arabidopsis had one or more representative ESTs. The LOC_Os10g41200 and AT5G47390 gene in rice and Arabidopsis had maximum number of ESTs, that is, 219 and 44, respectively. About 70\% of rice $M Y B$ genes and $80 \%$ of Arabidopsis $M Y B$ genes appeared to be highly expressed as evident from the availability of ESTs for these genes (Additional file 6: Table S6). Further, we assessed the expression levels of $M Y B$ genes under various abiotic stresses by PlantQTL-GE [69], GENEVESTIGATOR [70,71] and our previous microarray data experiment (E-MEXP-2401) with rice cv. Nagina 22 and IR64 under normal and drought conditions
(Additional file 7: Table S7). In our previous microarray data experiments, we found that 142 (92.26\%) $M Y B$ genes were expressed in seedlings of rice (Additional file 8: Figure S1), of which 92 genes were differentially regulated under drought stress. In IR64, 30 genes were upregulated ( $\geq 2.0$ fold) and 30 genes were down-regulated ( $\leq 2.0$ fold), while in Nagina 22, 22 genes were upregulated ( $\geq 2.0$ fold) and 19 genes were down-regulated ( $\leq 2.0$ fold) under drought stress. The exploration of PlantQTL-GE for rice MYBs showed that 14 (9.03\%) $O s M Y B$ genes were up-regulated under cold, drought and salt stress in rice, of which 10 are up-regulated under drought condition. These results suggest that large set of $M Y B$ genes may have a role in drought stress response in rice. Previous studies have shown that over-expression of $M Y B$ genes improved abiotic stress tolerance of rice and Arabidopsis $[24,72]$. In addition to these, we have identified additional $M Y B$ genes that are regulated by drought 


\begin{tabular}{|c|c|c|c|c|c|c|}
\hline $\begin{array}{l}\text { MEME-generated IUPAC } \\
\text { consensus motifs }\end{array}$ & $\begin{array}{l}\text { log likelihood } \\
\text { ratio (IIr) }\end{array}$ & E-Value & $\begin{array}{l}\text { Motif } \\
\text { conservation (\%) }\end{array}$ & $\begin{array}{l}\text { Motif description } \\
\text { in PLACE }\end{array}$ & Organism & Motif Logo \\
\hline \multicolumn{7}{|c|}{ Motifs from the common drought up-regulated promoters } \\
\hline YCYCCYCYYCYC & 439 & $1.1 \mathrm{e}-017$ & 83.33 & Novel motif & rice & \\
\hline GAGRRRRRRRR & 479 & $1.5 \mathrm{e}-018$ & 100 & Novel motif & rice & \\
\hline CGSCGSSSGYSG & 216 & $5.7 e-008$ & 31.48 & Novel motif & rice & \\
\hline AAWWAWAWAAWR & 431 & $9.4 \mathrm{e}-007$ & 88.88 & Novel motif & rice & \\
\hline YBYCTYYSYYKC & 376 & $2.1 \mathrm{e}-006$ & 72.22 & Novel motif & rice & \\
\hline \multicolumn{7}{|c|}{ Motifs from the common drought down-regulated promoters } \\
\hline CYCYYYCYCTSC & 373 & $1.3 \mathrm{e}-021$ & 100 & Novel motif & rice & \\
\hline СМКССКССКСС & 270 & $3.5 \mathrm{e}-011$ & 65.79 & Novel motif & rice & \\
\hline RRARAAAAARRA & 318 & $9.7 e-010$ & 84.21 & Novel motif & rice & \\
\hline GABRGARRGAG & 256 & $6.5 e-008$ & 63.15 & Novel motif & rice & \\
\hline TTWKTTWWTTTT & 334 & $3.5 e-007$ & 94.74 & CCA1 binding site & rice & \\
\hline \multicolumn{7}{|c|}{ Motifs from the group of R2R3-type MYB TFs } \\
\hline ARARARRRARAR & 733 & $2.3 e-034$ & 90.90 & Novel motif & rice & \\
\hline CYCTCYYYCYCY & 645 & $1.1 \mathrm{e}-034$ & 73.86 & Novel motif & rice & \\
\hline YCGCSSSCSCSS & 449 & $2.3 e-019$ & 46.59 & Novel motif & rice & \\
\hline TKWWTTTWTTTT & 585 & $1.3 \mathrm{e}-016$ & 70.45 & CCA1 binding site & rice & \\
\hline GGWGGHGGYGS & 370 & $1.6 \mathrm{e}-016$ & 37.5 & Novel motif & rice & \\
\hline ARARAARAAAAR & 1096 & $2.9 \mathrm{e}-041$ & 92.02 & Novel motif & AT & \\
\hline TYTYTYTYYYTY & 1015 & $5.1 \mathrm{e}-037$ & 81.88 & Novel motif & AT & \\
\hline RGAGAKAGAGRK & 378 & $7.5 e-011$ & 23.18 & Novel motif & AT & \\
\hline CAYMCAYRCRYR & 398 & $3.5 \mathrm{e}-001$ & 26.81 & Novel motif & AT & \\
\hline CTCWMWCTCTC & 180 & $8.9 \mathrm{e}+000$ & 10.14 & Novel motif & AT & \\
\hline
\end{tabular}

Figure 7 Conserved cis-motifs found in upstream promoter region of MYB genes in rice and Arabidopsis. a) Motifs from the promoter region of drought stress-regulated MYB genes in rice, $\mathbf{b})$ Motifs from the group of R2R3-MYB genes in both rice and Arabidopsis. 
and other stresses, and thus can be used as candidate genes for functional validation. The GENEVESTIGATOR analysis showed that 44.67, 41.12 and $56.85 \%$ AtMYB genes were down regulated and 47.21, 50.76 and $35.02 \%$ AtMYB genes were up regulated in cold, drought and salt stress, respectively (Additional file 9: Figure S2a, b and c, Additional file 10: Figure S3).

We analyzed expression patterns of 60 OsMYB and 21 AtMYB genes using QRT-PCR. These genes were selected based on phylogenetic analysis and one gene from each cluster was selected for expression analysis. Out of the 60 genes examined by QRT-PCR, 28 OsMYB genes were up-regulated ( $\geq 1.5$ fold change) under drought stress in rice cv. Nagina 22 (Figure 8). We also found that LOC_Os02g47744, LOC_Os12g41920 and LOC_Os06g19980 were highly up-regulated $(\geq 4$ fold change), indicating their potential role in drought stress. QRT-PCR analysis of $21 M Y B$ genes in Arabidopsis revealed that 7 AtMYB genes were up-regulated $(\geq 1.5$ fold changes) and another $7 A t M Y B$ genes were downregulated ( $\leq 1.5$ fold change) under drought stress (Figure 8).

\section{Tissue-specific expression}

In rice, a tissue breakdown of EST evidence for $M Y B$ genes was analyzed using the Rice Gene Expression Anatomy Viewer, MSU database [73,74]. In case of Arabidopsis, tissue-specific expressions of $M Y B$ genes were obtained from GENEVESTIGATOR tool [70,71]. The expression patterns of $M Y B$ genes in different tissues are listed in Additional file 11: Table S8. The results showed that large numbers of OsMYB genes (32.90\%) were highly expressed in the panicle, leaf and shoots (Additional file 12: Figure S4). EST frequency analysis suggested that OsMYB genes, LOC_Os02g34630, LOC_Os08g05510, LOC_Os01g74590, LOC_Os02g09480, LOC_Os09g36730, OsMYB4, LOC_Os10g41200 and LOC_Os01g13740 are highly expressed in flower, anther, endosperm, pistil, shoot, panicle, immature seed and whole plant, respectively. In case of leaves, we observed that three MYB genes, i.e., OsMYB48, LOC_Os06g40710 and LOC_Os10g41200 showed highest levels of expression. In Arabidopsis, the following $M Y B$ genes expressed at a very high level: AtMYBCDC5 in callus and seed; AT1G19000 in seedling and stem; AT1G74840 in root and root tip; AT1G26580 in flower, AtMYB91 in shoot, and AtMYB44 in pedicel and leaves. In wheat, TaMYB1 showed high expression in root, sheath and leaf, while TaMYB2 expression was highest in root and leaf, but at low in sheath [75]. TaMYB1 and TaMYB2 showed a very high sequence similarity with $A t M Y B 44$ and $O s M Y B 48$, respectively. Our analysis also revealed that these two MYBs are highly expressed in leaf as in case of wheat. These analyses will be useful in selecting candidate genes for functional analysis of their role in a specific tissue.

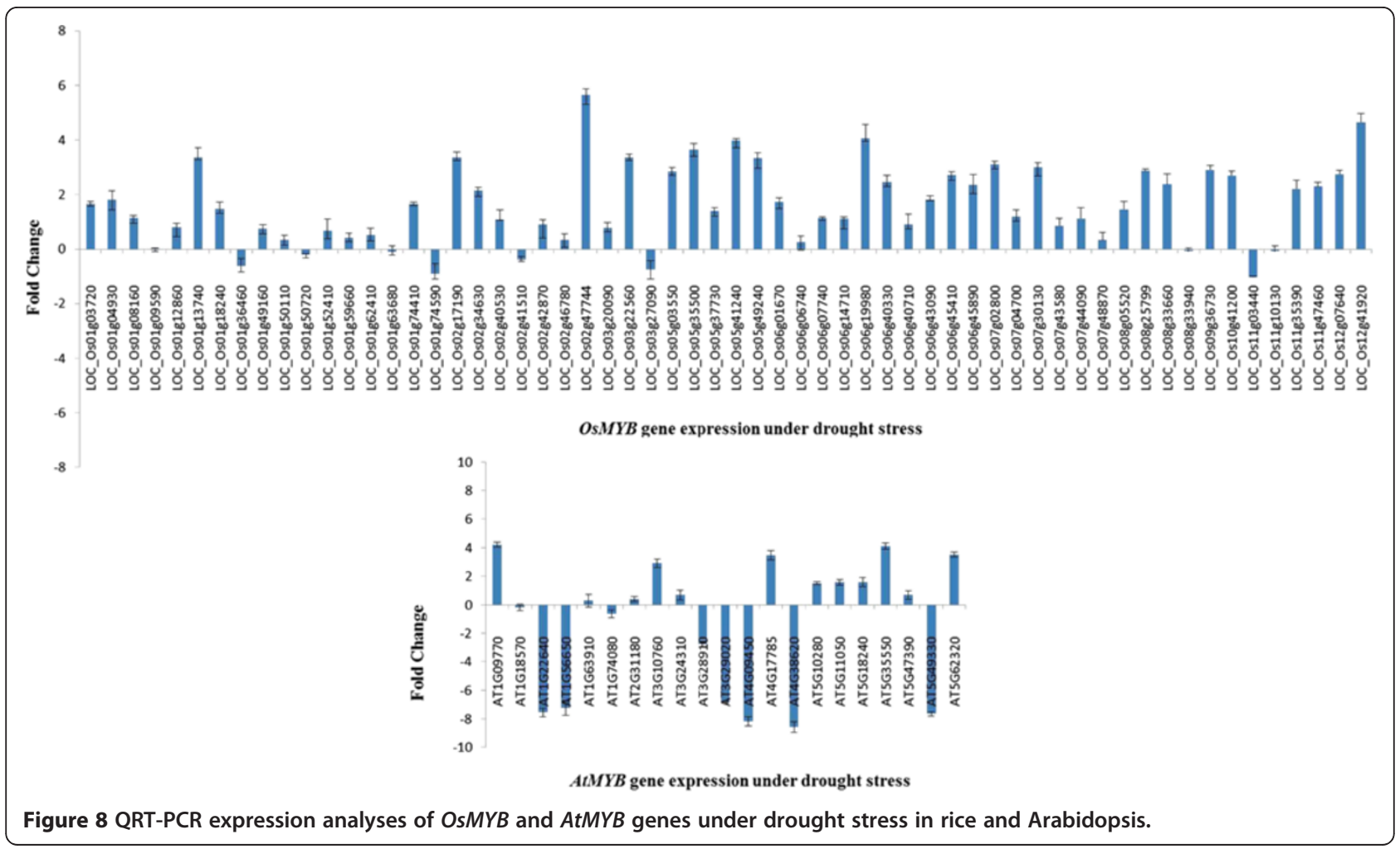




\section{Evolutionary relationship}

To understand the evolutionary relationship among $M Y B$ family genes, phylogenetic trees were constructed using the multiple sequence alignment of MYB proteins [76]. The tree revealed that tandem repeat and homologous pairs were grouped together into single clade with very strong bootstrap support (Additional file 13: Figure S5). These results further support gene duplication in rice and Arabidopsis during evolution which may allow functional diversification by adaptive protein structures [77]. It was also noticed that few "homologues pairs" (e.g. AT5G16600-AT3G02940 in Arabidopsis; LOC_Os12g07610LOC_Os12g07640 in rice) and "tandem repeat pairs" (e.g. AT3G12720-AT3G12730 in Arabidopsis; LOC_Os06g14700LOC_Os06g14710 in rice) were found in distinct clade, indicating that only few members had common ancestral origin that existed before the divergence of monocot and dicot. MYB proteins from rice and Arabidopsis with same number of MYB domains were grouped into a single clade. For instance, all the MYBs belonging to R1R2R3 family in both rice and Arabidopsis were clustered into single clade. Within the R2R3 clade, MYBs from rice and Arabidopsis were not found in distinct groups. These results suggest that significant expansion of R2R3-type $M Y B$ genes in plants occurred before the divergence of monocots and dicots, which in agreement with the previous studies $[4,62]$. Finally, we observed that two CDC5type and one 4-repeat MYB orthologs were clustered into single clade and might have been derived from an ancient paralog of widely distributed R2R3 MYB genes.

\section{Conclusions}

Our study provides genome-wide comparative analysis of MYB TF family gene organization, sequence diversity and expression pattern in rice and Arabidopsis. Structural analysis revealed that introns are highly conserved in the central region of the gene, and R2R3-type MYB proteins usually have two introns at conserved positions. Analysis of length and splicing of the intron/exon and their position in MYB domain suggested that introns were highly conserved within the same subfamily. Most of the MYB genes are present as duplicate genes in both rice and Arabidopsis. Phylogenetic analysis of rice and Arabidopsis MYB proteins showed that tandem repeat and homologous pair was grouped together into single clade. Consensus motif analysis of $1 \mathrm{~kb}$ upstream region of $M Y B$ gene ORFs led to the identification of conserved and over-represented cis-motifs in both rice and Arabidopsis. The comparative analysis of $M Y B$ genes in rice and Arabidopsis elucidated chromosomal location, gene structure and phylogenetic relationships, and expression analysis led to the identification of abiotic stress responsive and tissue-specific expression pattern of the selected $M Y B$ genes, suggesting functional diversification.
Our comprehensive analyses will help design experiments for functional validation of their precise role in plant development and stress responses.

\section{Methods}

Identification of $M Y B$ gene family in rice and Arabidopsis

To identify $M Y B$ transcription factor family genes, we searched and obtained genes annotated as MYB in MSU (release 5) for rice and TAIR (release 8) for Arabidopsis by using in-house PERL script along with careful manual inspection. The primary search disclosed 161 and 199 members annotated as "MYB" or "MYB-related genes" in MSU and TAIR database, respectively. We observed that some protein members lack MYB-DNA binding domain but still annotated as MYB protein family in MSU and TAIR database. We discarded these proteins based in the annotation in MSU (release 7) for rice and TAIR (release 10). Finally, we obtained 155 and 197 MYB genes in rice and Arabidopsis, respectively. The gene identifiers were assigned to each $O s M Y B$ and $A t M Y B$ genes to avoid confusion when multiple names are used for same gene. Uncharacterized $M Y B$ genes are denoted here by their locus id.

\section{MYB annotation}

To identify number of domains present in MYB protein we executed domain search by Conserved Domains Database [78] (http://www.ncbi.nlm.nih.gov/Structure/ cdd/cdd.shtml) and pfam database [79] (http://pfam. sanger.ac.uk/)with both local and global search strategy and expectation cut off ( $E$ value) 1.0 was set as the threshold for significance. Only significant domain found in rice and Arabidopsis MYB protein sequence were considered as a valid domain. To get more information about nature of the MYB protein, grand average of hydropathy (GRAVY), PI and the molecular weight were predicted by ProtParam tool available on Expert Protein Analysis System (ExPASy) proteomics server (http:// www.expasy.ch/tools/protparam.html). The subcellular localization of MYB proteins were predicted by Protein Localization Server (PLOC) (http://www.genome.jp/SIT/ plocdir/), Subcellular Localization Prediction of Eukaryotic Proteins (SubLoc V 1.0) (http://www.bioinfo.tsinghua.edu. cn/SubLoc/eu_predict.htm), SVM based server ESLpred (http://www.imtech.res.in/raghava/eslpred/submit.html), and ProtComp 9.0 server (http://linux1.softberry.com/ berry.phtml?topic=protcomppl\&group=programs\&subgroup= proloc). Further, species-specific localization prediction system was utilized for Arabidopsis (AtSubP, http:// bioinfo3.noble.org/AtSubP/) [57]. MYB protein function in term of their Gene Ontology (GO) was predicted by GO annotation search page available at MSU (http:// rice.plantbiology.msu.edu/downloads_gad.shtml) and TAIR (http://www.arabidopsis.org/tools/bulk/go/index.jsp) for 
rice and Arabidopsis, respectively. Localization consensus was predicted based on majority of result. The confidence level was acquired by assigning equal numeric value (e.g. one) to each general localization predictor and higher value to gene ontology (e.g. two) and species specific predictor (e.g. three).

\section{Identification of over-represented motifs}

We discovered over represented cis-motif consensus pattern in $1 \mathrm{~kb}$ upstream sequence from translational initiation codon of $M Y B$ genes in both rice and Arabidopsis using the Multiple Expectation maximization for Motif Elicitation analysis tool [80] (MEME version 4.1.0, http:// meme.sdsc.edu/meme/meme-intro.html). This program was used to search best 5 cis-motif consensus patterns of 8-12 bases width, with E-value $<0.01$, only on the forward strand of the input sequences. Motifs graph were plotted according to their position within the region using WebLogo tool (http://weblogo.berkeley.edu/logo. cgi). Discovered motifs were analyzed using PLACE [81] (http://www.dna.affrc.go.jp/PLACE/). Diurnal and circadian controlled $M Y B$ expression was explored from "Diurnal Version 2.0” (Mockler lab; http://diurnal.mocklerlab.org/).

\section{Phylogenetic analysis}

To generate the phylogenetic trees of $M Y B$ transcription factor family genes, multiple sequence alignment of MYB protein sequence were performed using COBALT program [82] (http://www.ncbi.nlm.nih.gov/tools/cobalt/). COBALT program automatically utilize information about bona fide proteins (i.e. MYB domains in this case) to execute multiple sequence alignment and build phylogenetic tree. The dendrogram were constructed with the following parameters; method-fast minimum evolution, max sequence difference-0.85, distance- grishin (protein).

\section{MYB localization, tandem repeat and duplication}

To map the gene loci on rice and Arabidopsis chromosomes pseudomolecules were used in MapChart (version 2.2) program [83] for rice and chromosome map tool [84] for Arabidopsis available on The Arabidopsis Information Resource (TAIR) database (http://www. arabidopsis.org/jsp/ChromosomeMap/tool.jsp). Tandem repeats were identified by manual visualization of rice and Arabidopsis physical map. Duplication or homologous pair genes were obtained by the segmental genome duplication segment (http://rice.plantbiology.msu. edu/segmental_dup/) and Arabidopsis Syntenic Pairs / Annotation Viewer (http://synteny.cnr.berkeley.edu/AtCNS/) in rice (distance $=500 \mathrm{~kb}$ ) and Arabidopsis, respectively. The tandem repeat and homologous pairs were aligned with the BLAST 2 SEQUENCE tool available on National Center on Biotechnology Information (NCBI) (http:// blast.ncbi.nlm.nih.gov/Blast.cgi/).

\section{Gene structure analysis}

To know more about intron / exon structure, $M Y B$ coding sequence (CDS) were aligned with their corresponding genomic sequences using spidey tool available on NCBI (http://www.ncbi.nlm.nih.gov/spidey/). To identify conserved intronless genes between rice and Arabidopsis, local protein blast (BLASTP) (http://www.molbiol. ox.ac.uk/analysis_tools/BLAST/BLAST_blastall.shtml) was performed for protein sequences of all predicted intronless genes in rice against all predicted intronless gene in Arabidopsis, and vice versa. Hits with 1e-6 or less were treated as conserved intronless genes and hits with $1 \mathrm{e}-10$ or less were treated as paralogs. The cutoff of sequence identity was considered as $\geq 20 \%$ over the $70 \%$ average query coverage.

\section{Expression analysis}

Expression support for each gene model is explored through gene expression evidence search page (http://rice. plantbiology.msu.edu/locus_expression_evidence.shtml) available at MSU for rice and GENEVESTIGATOR tool (https://www.genevestigator.com/) for Arabidopsis. $M Y B$ genes for which no ESTs were found, blast (BLASTP and TBLASTN) (http://blast.ncbi.nlm.nih.gov/Blast.cgi) search using NCBI databases was performed. Significant similarity of $M Y B$ genes with $M Y B$ genes of other plant species was searched. To measure the $M Y B$ expression level in abiotic stress plant QTLGE database was used (http://www.scbit. org/qtl2gene/new/) for rice and GENEVESTIGATOR tool (https://www.genevestigator.com/) for Arabidopsis. To identify tissue specific expression level of OsMYB genes in rice, highly expressed gene search (http://Rice. plantbiology.msu.edu/tissue.expression.shtml) available at MSU were used. For Arabidopsis, GENEVESTIGATOR tool (https://www.genevestigator.com/gv/user/gvLogin.jsp) was used.

\section{Plant materials and growth conditions}

The plant materials used were drought tolerant rice (Oryza sativa L. subsp. Indica) cv. Nagina 22 and Arabidopsis thaliana ecotype Columbia. The seeds were surface sterilized. Rice seeds were placed on absorbent cotton, which was soaked overnight in water and kept in medium size plastic trays. Arabidopsis seeds were germinated on MS-agar medium containing 1\% Sucrose and seven days old seedlings were transferred to soilrite for further growth. The rice and Arabidopsis seedlings were grown in a greenhouse under the photoperiod of $16 / 8 \mathrm{~h}$ light/dark cycle at $28^{\circ} \mathrm{C} \pm 1$ and $23^{\circ} \mathrm{C} \pm 1$, respectively.

\section{Drought stress treatment}

Drought was imposed to 3-weeks old rice seedlings [85] and 5-week-old Arabidopsis plants by withholding water till visible leaf rolling was observed. Control plants were 
irrigated with sufficient water. Plant water status was quantified by measuring relative water content of leaf. Control plants showed 96.89 and $97.49 \%$ RWC (relative water content), while stressed plants showed 64.86 and 65.2\% RWC in rice and Arabidopsis, respectively.

\section{Real-Time RT-PCR}

Total RNA from rice and Arabidopsis were isolated by TRIzol Reagent (Ambion) and treated with DNase (QIAGEN, GmbH). The first strand cDNA of rice and Arabidopsis was synthesized using Superscript III Kit (Invitrogen) from $1 \mu \mathrm{g}$ of total RNA according to manufacturer's protocol. Reverse transcription reaction was carried out at $44^{\circ} \mathrm{C}$ for $60 \mathrm{~min}$ followed by $92^{\circ} \mathrm{C}$ for $10 \mathrm{~min}$. Five ng of cDNA was used as template in a $20 \mu \mathrm{L}$ RT reaction mixture. Sixty three pairs of rice and 51 pairs of Arabidopsis gene specific primers were used to study expression of $M Y B$ transcription factor. Gene specific primers were designed using IDT PrimerQuest (http://www. idtdna.com/scitools/applications/primerquest/default.aspx). Ubiquitin and actin primers were used as an internal control in rice and Arabidopsis, respectively. The primer combinations used here for real-time RT-PCR analysis specifically amplified only one desired band. The dissociation curve testing was carried out for each primer pair showing only one melting temperature. The RT-PCR reactions were carried out at $95^{\circ} \mathrm{C}$ for $5 \mathrm{~min}$ followed by 40 cycles of $95^{\circ} \mathrm{C}$ for $15 \mathrm{~s}$ and $60^{\circ} \mathrm{C}$ for $30 \mathrm{~s}$ each by the method described previously by Dai et al., 2007 [24]. For qRT-PCR, QuantiFast SYBR Green PCR master mix (QIAGEN GmbH) was used according to manufacturer's instruction. The threshold cycles $\left(C_{\mathrm{T}}\right)$ of each test target were averaged for triplicate reactions, and the values were normalized according to the $C_{T}$ of the control products (Os-actin or Ubiquitin) in case of rice and Arabidopsis, respectively. $M Y B$ TFs expression data were normalized by subtracting the mean reference gene $\mathrm{CT}$ value from individual $\mathrm{CT}$ values of corresponding target genes $(\triangle \mathrm{CT})$. The fold change value was calculated using the expression, where $\Delta \Delta \mathrm{CT}$ represents difference between the $\Delta \mathrm{CT}$ condition of interest and $\triangle \mathrm{CT}$ control. The primer sets used to study the $M Y B$ TFs expression profile are given in the Additional file 14: Table S9.

\section{Additional files}

Additional file 1: Table S1. Nomenclature and classification of MYB TF family genes. Genome wide classification of MYB family genes including their characters such as GRAVY, PI, molecular weight and subcellular localization in rice and Arabidopsis.

Additional file 2: Table S2. Functional assignment and subcellular localization of MYB TF family proteins. Molecular functional annotation of MYB TF family by gene ontology enrichment analysis including their subcellular localization in rice and Arabidopsis.
Additional file 3: Table S3. Sequence alignment of intronless MYB genes. Sequence comparison between rice and Arabidopsis intronless genes to predict conserveness.

Additional file 4: Table S4. Density of Introns. Distribution of introns in the MYB domain and other region of MYB genes in rice and Arabidopsis.

Additional file 5: Table S5. Diurnal/circadian expression. MYB expression under diurnal/circadian conditions in rice and Arabidopsis.

Additional file 6: Table S6. Expression of MYB genes. Availability of fulllength complementary DNA (FL-cDNA) / expressed sequence tag (EST) consequent to MYB genes.

Additional file 7: Table S7. MYB regulation under abiotic stress. Expression analysis of MYB genes under abiotic stress conditions in rice and Arabidopsis by using publically available microarray data.

Additional file 8: Figure S1. MYB gene expression under drought stress in rice. Analysis of MYB gene expression under drought stress in rice. We obtained MYB expression from our previously published microarray gene expression experiments [64].

Additional file 9: Figure S2. MYB gene expression under abiotic stresses in Arabidopsis. MYB gene expression under cold (a), drought (b) and salt (c) stresses in Arabidopsis. GENEVESTIGATOR database was used to analyze the MYB gene expression levels.

Additional file 10: Figure S3. MYB expression profiling using heatmap in Arabidopsis. Expression profile of MYB gene using heatmap for cold, drought, and salt stress, fetched by GENEVESTIGATOR database.

Additional file 11: Table S8. Tissues specific MYB expression. Tissuespecific expression profiling of MYB genes in rice and Arabidopsis.

Additional file 12: Figure S4. MYB expression profiles of different tissues in rice. Tissue specific expression profile of MYB gene in rice examine by MSU database.

Additional file 13: Figure S5. Phylogenetic analysis of MYB proteins. Phylogenetic analysis of MYB proteins in both rice and Arabidopsis. The tree was constructed by using the multiple sequence alignment of bonafide MYB proteins.

Additional file 14: Table S9. Gene specific primers. List of gene specific primers used for QRT-PCR expression analysis of MYB genes in rice and Arabidopsis.

\section{Abbreviations}

MSU: Michigan State University; TAIR: The Arabidopsis Information Resource; PERL: Practical Extraction and Report Language; GO: Gene Ontology; BLAST: Basic Local Alignment Search Tool; MEME: Multiple Expectation Maximization for Motif Elicitation; EST: Expressed Sequence Tag; NCBI: National Center for Biotechnology Information; GEO: Gene Expression Omnibus; QRT-PCR: Quantitative Reverse Transcription Polymerase Chain Reaction.

\section{Competing interests}

The authors declare that they have no competing interests.

\section{Authors' contributions}

AK performed all the bioinformatics analysis, including large-scale sequence analysis and mapping, and drafted the manuscript; SS helped in bioinformatics analysis, data mining and management; SKL conceived the idea of identification of MYB TF's and designed the study; RR carried out all the wet-lab experiments; VC and KCB guided in the design of the study and drafting the manuscript. All authors read and approved the final manuscript.

\section{Acknowledgements}

We thank Indian Council of Agricultural Research (ICAR) for supporting this work through the ICAR-sponsored Network Project on Transgenics in Crops (NPTC) and National Initiative on Climate Resilient Agriculture (NICRA). SKL gratefully acknowledge University Grants Commission (UGC) and Council of Scientific and Industrial Research (CSIR) for CSIR-UGC Junior and Senior Research Fellowship Grant. SS and RR acknowledge the senior research and research associate fellowship grant by Department of Biotechnology (DBT), Govt. of India, respectively. We thank Cathie Martin, John Innes Centre, 
Norwich Research Park, Colney, Norwich, UK, for her valuable suggestions on the data analysis and manuscript

\section{Author details}

${ }^{1}$ National Research Centre on Plant Biotechnology, Indian Agricultural Research Institute, New Delhi 110012, India. ${ }^{2}$ National Bureau of Plant Genetic Resources, Indian Agricultural Research Institute Campus, New Delhi 110012, India. ${ }^{3}$ Department of Biology, University of Massachusetts, Amherst, MA 01003, USA. ${ }^{4}$ Department of Biotechnology, Assam University, Silchar, Assam 788011, India. ${ }^{5}$ Division of Plant Physiology, Indian Agricultural Research Institute, New Delhi 110012, India.

Received: 14 February 2012 Accepted: 1 October 2012

Published: 10 October 2012

\section{References}

1. Ptashne M: How eukaryotic transcriptional activators work. Nature 1988 335:683-689.

2. Klempnauer KH, Gonda TJ, Bishop JM: Nucleotide sequence of the retroviral leukemia gene v-myb and its cellular progenitor c-MYB: the architecture of a transduced oncogene. Cell 1982, 31:453-463.

3. Weston K: Myb proteins in life, death and differentiation. Curr Opin Genet Dev 1998, 8:76-81

4. Lipsick JS: One billion years of Myb. Oncogene 1996, 13:223-235.

5. Paz-Ares J, Ghosal D, Wienand U, Peterson P, Saedler $H$ : The regulatory $\mathrm{c} 1$ locus of Zea mays encodes a protein with homology to MYB oncogene products and with structural similarities to transcriptional activators. EMBO J 1987, 6:3553-3558.

6. Riechmann JL, Heard J, Martin G, Reuber L, Jiang C, Keddie J, Adam L, Pineda O, Ratcliffe OJ, Samaha RR, Creelman R, Pilgrim M, Broun P, Zhang JZ, Ghandehari D, Sherman BK, Yu G: Arabidopsis transcription factors: genome-wide comparative analysis among eukaryotes. Science 2000, 290:2105-2110.

7. Martin C, Paz-Ares J: MYB transcription factors in plants. Trends Genet 1997 13:67-73.

8. Kranz H, Scholz K, Weisshaar B: c-MYB oncogene-like genes encoding three MYB repeats occur in all major plant lineage. Plant $J 2000$, 21:231-235.

9. Yanhui $C$, Xiaoyuan $Y$, Kun $H$, Meihua L, Jigang L, Zhaofeng G, Zhiqiang $L$, Yunfei Z, Xiaoxiao W, Xiaoming Q, Yunping S, Li Z, Xiaohui D, Jingchu L, Xing-Wang D, Zhangliang C, Hongya G, Li-Jia Q: The MYB transcription factor superfamily of Arabidopsis: expression analysis and phylogenetic comparison with the rice MYB family. Plant Mol Biol 2006, 60:107-124.

10. Pasquali G, Biricolti S, Locatelli F, Baldoni E, Mattana M: OsMYB4 expression improves adaptive responses to drought and cold stress in transgenic apples. Plant Cell Rep 2008, 27:1677-1686.

11. Li-Jia Q, Zhu Y-X: Transcription factor families in Arabidopsis: major progress and outstanding issues for future research. Curr Opin Plant Biol 2006, 9:544-549.

12. Feller A, Machemer K, Braun EL, Grotewold E: Evolutionary and comparative analysis of MYB and bHLH plant transcription factors. Plant J 2011, 66(1):94-116

13. Kanei-Ishii C, Sarai A, Sawazaki T, Nakagoshi H, He DN, Ogata K, Nishimura Y, Ishii S: The tryptophan cluster: a hypothetical structure of the DNA-binding domain of the myb protooncogene product. J Biol Chem 1990, 265:19990-19995.

14. Ogata K, Morikawa S, Nakamura H, Sekikawa A, Inoue T, Kanai H, Sarai A Ishii S, Nishimura Y: Solution structure of a specific DNA complex of the MYB DNA-binding domain with cooperative recognition helices. Cell 1994, 79:639-648.

15. Dubos C, Stracke R, Grotewold E, Weisshaar B, Martin C, Lepiniec L: MYB transcription factors in Arabidopsis. Trends Plant Sci 2010, 15:1360-1385.

16. Jiang C, Gu J, Chopra S, Gu X, Peterson T: Ordered origin of the typical two- and three-repeat Myb genes. Gene 2004, 326:13-22.

17. Rosinski JA, Atchley WR: Molecular evolution of the Myb family of transcription factors: evidence for polyphyletic origin. J Mol Evol 1998, 46:74-83.

18. Allan AC, Hellens RP, Laing WA: MYB transcription factors that colour our fruit. Cell 2008, 13:99-102.

19. Cominelli $E$, Tonelli $C$ : A new role for plant R2R3-MYB transcription factors in cell cycle regulation. Cell Res 2009, 19:1231-1232.
20. Agarwal $M$, Hao $Y$, Kapoor $A$, Dong $C H$, Fujii $H$, Zheng $X$, Zhu JK: A R2R3 type MYB transcription factor is involved in the cold regulation of $C B F$ genes and in acquired freezing tolerance. J Biol Chem 2006 281:37636-37645.

21. Ma Q, Dai X, Xu Y, Guo J, Liu Y, Chen N, Xiao J, Zhang D, Xu Z, Zhang X, Chong K: Enhanced tolerance to chilling stress in OsMYB3R-2 transgenic rice is mediated by alteration in cell cycle and ectopic expression of stress genes. Plant Physiol 2009, 150:244-256.

22. Vannini C, Locatelli F, Bracale M, Magnani E, Marsoni M, Osnato M, Mattana M, Baldoni E, Coraggio I: Overexpression of the rice OsMYB4 gene increases chilling and freezing tolerance of Arabidopsis thaliana plants. Plant J 2004, 37:115-127.

23. Seo PJ, Xiang F, Qiao M, Park JY, Lee YN, Kim SG, Lee YH, Park WJ, Park CM: The MYB96 transcription factor mediates abscisic acid signaling during drought stress response in Arabidopsis. Plant Physio/ 2009, 151:275-289.

24. Ding Z, Li S, An X, Liu X, Qin H, Wang D: Transgenic expression of MYB15 confers enhanced sensitivity to abscisic acid and improved drought tolerance in Arabidopsis thaliana. Cell Res 2008, 18:1047-1060.

25. Ito M, Araki S, Matsunaga S, Itoh T, Nishihama R, Machida Y, Doonan JH, Watanabe A: G2/M-phase-specific transcription during the plant cell cycle is mediated by c-MYB-like transcription factors. Plant Cell 2001, 13:1891-1905

26. Araki S, Ito M, Soyano T, Nishihama R, Machida Y: Mitotic cyclins stimulate the activity of c-MYB-like factors for transactivation of G2/M phasespecific genes in tobacco. J Biol Chem 2004, 279:32979-32988.

27. Dai $X, X u Y, M a ~ Q, X u$ W, Wang T, Xue Y, Chong K: Overexpression of an R1R2R3 MYB Gene, OSMYB3R-2, increases tolerance to freezing, drought, and salt stress in transgenic Arabidopsis. Plant Physiol 2007, 143:1739-1751.

28. Haga N, Kato K, Murase M, Araki S, Kubo M, Demura T, Suzuki K, Muller I, Voss $U$, Jurgens $G$, Ito M: R1R2R3-MYB proteins positively regulate cytokinesis through activation of KNOLLE transcription in Arabidopsis thaliana. Development 2007, 134:1101-1110

29. Allen RS, Li J, Stalhe Ml, Dubroue A, Gubler F, Millar A: Genetic analysis reveals functional redundancy and the major target genes of the Arabidopsis miR159 family. Proc. Natl. Acad. Sci. USA 2007. 104:16371-16376.

30. Addo-Quaye C, Eshoo TW, Bartel DP, Axtell MJ: Endogenous siRNA and miRNA targets identified by sequencing of the Arabidopsis degradome. Current Biol. 2008, 18:758-762.

31. Stracke R, Werber M, Weisshaar B: The R2R3-MYB gene family in Arabidopsis thaliana. Curr Opin Plant Biol 2001, 4:447-456.

32. Shinozaki K, Yamaguchi-Shinozaki K, Urao T, Koizumi M: Nucleotide sequence of a gene from Arabidopsis thaliana encoding a MYB homologue. Plant Mol Biol 1992, 19:493-499.

33. Romero I, Fuertes A, Benito MJ, Malpical JM, Leyva A, Paz-Ares J: More than 80 R2R3-MYB regulatory genes in the genome of Arabidopsis thaliana. Plant J 1998, 14:273-284

34. Kranz HD, Denekamp M, Greco R, Jin H, Leyva A, Meissner RC, Petroni K, Urzainqui A, Bevan M, Martin C: Towards functional characterization of the members of the R2R3-MYB gene family from Arabidopsis thaliana. Plant J 1998, 16:263-276.

35. Karamysheva ZN, Surovtseva YV, Vespa L, Shakirov EV, Shippen DE: A C-terminal Myb extension domain defines a novel family of doublestrand telomeric DNA-binding proteins in Arabidopsis. J Biol Chem 2004 279:47799-47807.

36. Jin H, Martin C: Multifunctionality and diversity within the plant MYB-gene family. Plant Mol Biol 1999, 41:577-585.

37. Lu SX, Knowles SM, Andronis C, Ong MS, Tobin EM: CIRCADIAN CLOCK ASSOCIATED1 and LATE ELONGATED HYPOCOTYL function synergistically in the circadian clock of Arabidopsis. Plant Physiol 2009, 150:834-843.

38. Simon M: Distinct and overlapping roles of single-repeat MYB genes in root epidennal patterning. Dev Biol 2007, 311:566-578.

39. Dubos C: MYBL2 is a new regulator of flavonoid biosynthesis in Arabidopsis thaliana. Plant J 2008, 55:940-953.

40. Matsui K: AtMYBL2, a protein with a single MYB domain, act as negative regulator of anthocyanin biosynthesis in Arabidopsis. Plant J 2008, 55:954-967.

41. Pesch M, Hulskamp M: One, two, thee. Models for trichome patterning in Arabidopsis. Curr. Opin. Plant Biol. 2009, 12:587-592. 
42. Kyte J, Doolittle RF: A simple method for displaying the hydropathic character of a protein. J Mol Biol 1982, 157:105-132

43. Ramachandran S, Hiratsuka K, Chua NH: Transcription factors in plant growth and development. Curr Opin Genet Dev 1994, 4(5):642-646.

44. Borevitz JO, Xia Y, Blount J, Dixon RA, Lamb C: Activation tagging identifies a conserved MYB regulator of phenylpropanoid biosynthesis. Plant Cell 2000, 12:2383-2394.

45. Nesi $N$, Jond C, Debeaujon I, Caboche M, Lepiniec L: The Arabidopsis TT2 gene encodes an R2R3 MYB domain protein that acts as a key determinant for proanthocyanidin accumulation in developing seed. Plant Cell 2001, 13:2099-2114.

46. Baudry A, Heim MA, Dubreucq B, Caboche M, Weisshaar B, Lepiniec L: TT2, TT8, and TTG1 synergistically specify the expression of BANYULS and proanthocyanidin biosynthesis in Arabidopsis thaliana. Plant J 2004, 39:366-380.

47. Lee MM, Schiefelbein J: WEREWOLF, a MYB-related protein in Arabidopsis, is a position-dependent regulator of epidermal cell patterning. Cell 1999, 99:473-483.

48. Lee MM, Schiefelbein J: Developmentally distinct MYB genes encode functionally equivalent proteins in Arabidopsis. Development 2001 12:1539-1546.

49. Higginson T, Li SF, Parish RW: AtMYB103 regulates tapetum and trichome development in Arabidopsis thaliana. Plant J 2003, 35:177-192.

50. Ito M: Conservation and diversification of three-repeat MYB transcription factor in plants. J Plant Res 2005, 118:61-69.

51. Jin H, Cominelli E, Bailey P, Parr A, Mehrtens F, Jones J, Tonelli C, Weisshaar B, Martin C: Transcriptional repression by AtMYB4 controls production of UV-protecting sunscreens in Arabidopsis. EMBO J 2000, 19:6150-6161.

52. Hemm MR, Herrmann KM, Chapple C: AtMYB4: a transcription factor general in the battle against UV. Trends Plant Sci 2001, 6:135-136.

53. Walker JC: Receptor-like protein kinase genes of Arabidopsis thaliana. Plant J 1993, 3:451-456.

54. Bender J, Fink GR: A MYB homologue, ATR1, activates tryptophan gene expression in Arabidopsis. Proc. Natl. Acad. Sci. USA 1998, 95:5655-5660.

55. Kim JH, Lee BW, Schroeder FC, Jander G: Identification of indoleglucosinolate breakdown products with antifeedant effects on Myzus persicae (green peach aphid). Plant J 2008, 54:1015-1026.

56. Kirik V, Lee MM, Wester K, Herrmann U, Zheng Z, Oppenheimer D, Schiefelbein J, Hulskamp M: Functional diversification of MYB23 and GL1 genes in trichome morphogenesis and initiation. Development 2005, 132:1477-1485.

57. Kaundal R, Saini R, Zhao PX: Combining Machine Learning and Homologybased Approaches to Accurately Predict Subcellular Localization in Arabidopsis. Plant Physiol 2010, 154(1):36-54.

58. Jain M, Khurana P, Tyagi AK, Khurana JP: Genome-wide analysis of intronless genes in rice and Arabidopsis. Funct Integr Genomics 2008 8:69-78

59. Li J, Li X, Guo L, Lu F, Feng X, He K, Wei L, Chen Z, Qu LJ, Gu H: A subgroup of MYB transcription factor genes undergoes highly conserved alternative splicing in Arabidopsis and rice. J Exp Bot 2006, 57(6):1263-1273.

60. Blanc G, Wolfe KH: Functional divergence of duplicated genes formed by polyploidy during Arabidopsis evolution. Plant Cell 2004, 16:1679-1691.

61. Thomas BC, Pedersen B, Freeling M: Following tetraploidy in an Arabidopsis ancestor, genes were removed preferentially from one homeolog leaving clusters enriched in dose-sensitive genes. Genome Res 2006, 16:934-946.

62. Rabinowicz PD, Braun EL, Wolfe AD, Bowen B, Grotewold E: Maize R2R3 $M Y B$ genes: sequence analysis reveals amplification in higher plants. Genetics 1999, 153:427-444

63. Braun EL, Grotewold E: Diversification of the R2R3 MYB gene family and the segmental allotetraploid origin of the maize genome. Maize Genet Coop News/ 1999, 73:26-27.

64. Lenka SK, Katiyar A, Chinnusamy V, Bansal KC: Comparative analysis of drought responsive transcriptome in Indica rice genotypes with contrasting drought tolerance. Plant Biotechnol J 2011, 9(3):315-327.

65. Lenka SK, Lohia B, Kumar A, Chinnusamy V, Bansal KC: Genome-wide targeted prediction of $A B A$ responsive genes in rice based on over-represented cismotif in co-expressed genes. Plant Mol Biol 2009, 69:261-271.
66. Wang ZY, Kenigsbuch D, Sun L, Harel E, Ong MS, Tobin EM: A Myb-related transcription factor is involved in the phytochrome regulation of an Arabidopsis Lhcb gene. Plant Cell 2007, 9:491-507.

67. Mockler TC, Michael TP, Priest HD, Shen R, Sullivan CM, Givan SA, McEntee C, Kay S, Chory J: Diurnal and circadian expression profiling, model-based pattern matching and promoter analysis. Cold Spring Harb Symp Quant Biol 2007, 72:353-363.

68. Boguski MS, Lowe TM, Tolstoshev CM: dbEST-database for "expressed sequence tags". Nat Genet 1993, 4:332-333.

69. Zeng H, Luo L, Zhang W, Zhou J, Li Z, Liu H, Zhu T, Feng X, Zhong Y: PlantQTL-GE: a database system for identifying candidate genes in rice and Arabidopsis by gene expression and QTL information. Nucleic Acids Res 2007, 35:D879-D882

70. Zimmermann P, Hoffmann MH, Hennig L, Gruissem W: GENEVESTIGATOR. Arabidopsis microarray database and analysis toolbox. Plant Physiol 2004 136:2621-2632.

71. Hruz T, Laule O, Szabo G, Wessendorp F, Bleuler S, Oertle L, Widmayer P, Gruissem W, Zimmermann P: Genevestigator V3: A Reference Expression Database for the Meta-Analysis of Transcriptomes. Adv Bioinformatics 2008, 2008:420747.

72. Yang A, Dai X, Zhang WH: A R2R3-type MYB gene, OsMYB2, is involved in salt, cold, and dehydration tolerance in rice. J Exp Bot 2012, 63(7):2541-56.

73. Yuan Q, Ouyang S, Wang A, Zhu W, Maiti R, Lin H, Hamilton J, Haas B, Sultana R, Cheung F, Wortman J, Buell CR: The institute for genomic research Osa1 rice genome annotation database. Plant Physiol 2005, 138:18-26.

74. Ouyang S, Zhu W, Hamilton J, Lin H, Campbell M, Childs K, Thibaud-Nissen F, Malek RL, Lee Y, Zheng L, Orvis J, Haas B, Wortman J, Buell CR: The TIGR rice Ggenome annotation resource: improvements and new features. Nucleic Acids Res 2007, 35:D883-D887.

75. Chen R, Ni Z, Nie X, Qin Y, Dong G, Sun Q: Isolation and characterization of genes encoding Myb transcription factor in wheat (Triticum aestivem L.). Plant Sci 2005, 169:1146-1154

76. Larkin MA, Blackshields G, Brown NP, Chenna R, McGettigan PA, McWilliam $H$, Valentin F, Wallace IM, Wilm A, Lopez R, Thompson JD, Gibson TJ, Higgins DG: Clustal W and Clustal X version 2.0. Bioinformatics 2007, 23:2947-2948

77. Li J, Clegg MT, Jiang T: Evolutionary Dynamics of the DNA-Binding Domains in Putative R2R3-MYB Genes Identified from Rice Subspecies indica and japonica Genomes. Plant Physiol 2004, 135:575-585.

78. Marchler-Bauer A, Lu S, Anderson JB, Chitsaz F, Derbyshire MK, Deweese-Scott C, Fong JH, Geer LY, Geer RC, Gonzales NR, Gwadz M, Hurwitz DI, Jackson JD, Ke Z, Lanczycki CJ, Lu F, Marchler GH, Mullokandov M, Omelchenko MV, Robertson CL, Song JS, Thanki N, Yamashita RA, Zhang D, Zhang N, Zheng C, Bryant SH: CDD: a Conserved Domain Database for the functional annotation of proteins. Nucleic Acids Res 2011, 39:D225-D229.

79. Finn RD, Tate J, Mistry J, Coggill PC, Sammut JS, Hotz HR, Ceric G, Forslund K, Eddy SR, Sonnhammer EL, Bateman A: The Pfam protein families database. Nucleic Acids Res 2008, 36:D281-D288.

80. Bailey TL, Williams N, Misleh C, Li WW: MEME: discovering and analyzing DNA and protein sequence motifs. Nucleic Acids Res 2006, 34:W369-W373.

81. Higo K, Ugawa $Y$, Iwamoto M, Korenaga T: Plant cis-acting regulatory DNA elements (PLACE) database. Nucleic Acids Res 1999, 27:297-300.

82. Papadopoulos JS, Agarwala R: COBALT: constraint-based alignment tool for multiple protein sequences. Bioinformatics 2007, 23:1073-1079.

83. Voorrips RE: MapChart: Software for the graphical presentation of linkage maps and QTLs. J Hered 2002, 93(1):77-78.

84. Poole RL: The TAIR database. Methods Mol Biol 2007, 406:179-212.

85. Salekdeh GH, Siopongco J, Wade LJ, Ghareyazie B, Bennett B: Proteomic analysis of rice leaves during drought stress and recovery. Proteomics 2002, 2:1131-1145.

doi:10.1186/1471-2164-13-544

Cite this article as: Katiyar et al:: Genome-wide classification and expression analysis of MYB transcription factor families in rice and Arabidopsis. BMC Genomics 2012 13:544. 